\title{
The Scaling of Broadband Shock-Associated Noise with Increasing Temperature
}

\author{
Steven A. E. Miller* \\ The National Aeronautics and Space Administration
}

\begin{abstract}
A physical explanation for the saturation of broadband shock-associated noise (BBSAN) intensity with increasing jet stagnation temperature has eluded investigators. An explanation is proposed for this phenomenon with the use of an acoustic analogy. For this purpose the acoustic analogy of Morris and Miller is examined. To isolate the relevant physics, the scaling of BBSAN at the peak intensity level at the sideline $(\psi=90$ degrees $)$ observer location is examined. Scaling terms are isolated from the acoustic analogy and the result is compared using a convergent nozzle with the experiments of Bridges and Brown and using a convergent-divergent nozzle with the experiments of Kuo, McLaughlin, and Morris at four nozzle pressure ratios in increments of total temperature ratios from one to four. The equivalent source within the framework of the acoustic analogy for BBSAN is based on local field quantities at shock wave shear layer interactions. The equivalent source combined with accurate calculations of the propagation of sound through the jet shear layer, using an adjoint vector Green's function solver of the linearized Euler equations, allows for predictions that retain the scaling with respect to stagnation pressure and allows for the accurate saturation of BBSAN with increasing stagnation temperature. This is a minor change to the source model relative to the previously developed models. The full development of the scaling term is shown. The sources and vector Green's function solver are informed by steady Reynolds-Averaged Navier-Stokes solutions. These solutions are examined as a function of stagnation temperature at the first shock wave shear layer interaction. It is discovered that saturation of BBSAN with increasing jet stagnation temperature occurs due to a balance between the amplification of the sound propagation through the shear layer and the source term scaling.
\end{abstract}

\section{Nomenclature}

$\begin{array}{ll}a_{m n} & \text { Coefficients of anisotropic turbulence } \\ c & \text { Speed of sound } \\ D & \text { Nozzle exit diameter } \\ f_{i} & \text { Unsteady force per unit volume associated with velocity fluctuations } \\ g & \text { Adjoint Green's function of the convective wave equation in the frequency domain } \\ g_{l} & \text { Green's function of Lilley's equation } \\ H_{i}^{(j)} & \text { Hankel function of kind } j \text { and order } i \\ J_{i} & \text { Bessel function of the first kind and order } i \\ K & \text { Turbulent kinetic energy } \\ l & \text { Integral length scale in the streamwise direction } \\ l_{\perp} & \text { Integral length scale in the cross-stream direction } \\ M & \text { Mach number } \\ M_{d} & \text { Design Mach number } \\ M_{j} & \text { Fully expanded Mach number } \\ m & \text { Azimuthal mode number } \\ N P R & \text { Nozzle pressure ratio }\end{array}$

\footnotetext{
* Research Aerospace Engineer, Aeroacoustics Branch, NASA Langley Research Center, 2 N. Dryden St. MS 461, Hampton, VA, 23681, USA, AIAA Member, s.miller@nasa.gov
} 


\begin{tabular}{|c|c|}
\hline$p$ & Pressure \\
\hline$\tilde{p}_{s}$ & Fourier transform of the shock pressure perturbation in the streamwise direction \\
\hline$R$ & Distance from nozzle exit to observer \\
\hline$R_{O}$ & Radial location between the inner and outer solutions of the Green's function solver \\
\hline$R_{n m}$ & Two-point cross-correlation of the turbulent velocity fluctuations \\
\hline$R_{n m}^{v}$ & Two-point cross-correlation of $f_{i}^{v}$ \\
\hline$r$ & Radial direction \\
\hline$S$ & Spectral density \\
\hline$S t$ & Strouhal number \\
\hline$T_{c f}$ & Temperature correction factor \\
\hline$T T R$ & Total temperature ratio \\
\hline$t$ & Time \\
\hline$u$ & Streamwise velocity component \\
\hline$v$ & Radial velocity component \\
\hline$v_{g i}^{n}$ & Component of the vector Green's function of the linearized Euler equations \\
\hline$x^{9 \imath}$ & Streamwise direction \\
\hline$\underline{x}=\underline{x}(x, y, z)$ & Vector observer position \\
\hline $\bar{y}=\bar{y}(x, y, z)$ & Vector source position from the primary nozzle exit \\
\hline & Off-design parameter \\
\hline$\gamma$ & Ratio of specific heats \\
\hline$\delta$ & Dirac delta function \\
\hline$\epsilon$ & Dissipation rate of turbulent kinetic energy \\
\hline$\eta=\eta(\xi, \eta, \sigma)$ & Vector between two source locations \\
\hline $\bar{\lambda}-\overline{-}$ & Parameter equal to $\omega \sin \theta / c_{\infty}$ \\
\hline$\pi_{g}^{n}$ & Vector Green's function of the linearized Euler equations \\
\hline$\rho$ & Density \\
\hline$\tau$ & Retarded time \\
\hline$\tau_{s}$ & Turbulent integral time scale \\
\hline$\phi$ & Azimuthal angle \\
\hline$\Psi$ & Observer angle from the nozzle inlet axis \\
\hline$\omega$ & Radian frequency \\
\hline \multicolumn{2}{|l|}{ Subscript } \\
\hline$j$ & Jet fully expanded quantity \\
\hline$s$ & Property of the shocks or turbulence scale \\
\hline$\infty$ & Ambient value \\
\hline
\end{tabular}

\section{Introduction}

Aircraft, rockets, and many other vehicles use jet engines as their means of propulsion. The noise produced by jet plumes is often problematic both physically ${ }^{1}$ and psychologically. ${ }^{2}$ For example, civilian and military aircraft operating in the vicinity of airports can be an annoyance to the community ${ }^{3}$ due to jet noise. During take-off, noise from the jet exhaust often dominates other aircraft noise sources. ${ }^{4}$ High speed supersonic jet flows cause hearing loss for military personnel during carrier landing and take-off and on military practice fields. ${ }^{5}$ If aircraft cruise a component of the cabin noise is due to jet noise. The loading induced by jet noise can cause sonic fatigue on aircraft and rocket engines. ${ }^{1}$ Rockets used to place delicate scientific or communication satellites in orbit often undergo large amounts of vibration on lift-off, which is in part due to upstream traveling acoustic waves caused by the jet plume. ${ }^{6,7}$ Clearly, there is a need for fundamental understanding, prediction, and mitigation of noise from jets at all velocity and temperature regimes.

There are multiple source mechanisms of jet noise. Their description and uniqueness is a subject of debate within the aeroacoustics community. Noise spectra can be observed in the far-field as shown in Fig. 1 from an off-design singlestream supersonic jet. The $x$-axis represents non-dimensional frequency as Strouhal number, $S t$, which is frequency normalized by the fully expanded jet velocity, $u_{j}$, and the fully expanded jet diameter, $D_{j}$. The $y$-axis represents the Sound Pressure Level (SPL) per unit $S t$ referenced to twenty micro 
Pascals. Two sources of jet noise are considered, the first is mixing noise and the second is shock-associated noise. Shock-associated noise consists of discrete tones often called 'screech,' first observed and described by Powell, ${ }^{8}$ and a broadband component called broadband shock-associated noise (BBSAN) which was first extensively modeled and studied by Harper-Bourne and Fisher. ${ }^{9}$ These noise components are labeled in the spectra shown in Fig. 1. The observer angle $\psi$ is measured from the upstream axis of the jet centerline to the observer in the far-field about the nozzle exit plane. The distance from the nozzle exit to the observer is $R / D=100$, where $D$ is the nozzle diameter. Turbulent mixing noise occurs at all jet Mach numbers and temperatures. It is a unique noise source that occurs due to the turbulence present in the jet plume. The exact mechanism of how sound waves are produced by turbulence is not fully understood by the community. The difficulty of understanding how noise is produced by turbulence cannot be understated, as turbulence is a highly chaotic process that is extremely sensitive to initial and boundary conditions. However, the acoustic analogy has given more physical insight into the sound generation process than any other approach, and has yielded valuable mathematical descriptions that have not been obtained by any other methodology.

Turbulent mixing noise is broadband in nature and dominant in the downstream quadrant $(\psi=150$ deg. in Fig. 1) of the jet relative to the nozzle exit. Turbulent mixing noise has been widely studied, and there is a large amount of literature within this classical subject of fluid dynamics. For excellent overviews of jet noise consult Ffowcs-Williams ${ }^{10},{ }^{11}$ or Goldstein, ${ }^{12}$ and specifically for supersonic jet noise consult Tam. ${ }^{13}$

Shock-associated jet noise can be observed in Fig. 1 as a discrete tone and multiple broadband peaks. The tonal and broadband noise are due to shock wave shear layer interactions and are highly related. The discrete tones do not always appear in experimental measurements. BBSAN and screech tones occur when the jet Mach number is greater than unity and the nozzle is operating off-design. A nozzle operates off-design when the static pressure in the ambient environment is not equal to the fully expanded pressure of the jet plume. When this pressure imbalance occurs a system of shock and Prandtl-Meyer expansion waves are contained within the shear layer of the jet. This system, called a shock cell structure, can be seen in the schlieren image produced by Zaman et al. ${ }^{14}$ in Fig. 2. Over-expanded jets result in an initial oblique shock wave and under-expanded jets result in an initial expansion wave attached at the nozzle lip.

Large-scale coherent turbulent structures convect at high speeds in the jet shear layer. These large-scale coherent structures are responsible for Mach wave radiation which is highly directional in the downstream direction and is classified as a type of mixing noise. Examine Tam ${ }^{15}$ for details on Mach wave radiation. The large-scale coherent turbulent structures interact with the shock cell structure in the jet shear layer. Each interaction of turbulence with individual oblique shock waves is a source that contributes to BBSAN. The noise combines constructively or destructively in the far-field to produce the broadband humps that can be seen in Fig. 1. The shock wave shear layer interaction can also cause a feedback loop that produces discrete tones that are often many times higher in magnitude than other noise components, and often at multiple discrete frequencies. These tones are caused by downstream traveling disturbances that are reflected by the oblique shock waves and then propagate upstream after which the process repeats. Screech tones are often not present in jet flows from modern engines because complicated geometry disturbs the feedback mechanism. Screech is often eliminated or minimized by the addition of heating. For an excellent description of screech tones see Raman. ${ }^{16}$ Screech tones, turbulent mixing noise, and other source mechanisms are not considered further in this paper, and only BBSAN is considered. However, screech tones have a large effect on BBSAN which will be illustrated later.

BBSAN is characterized by multiple broad lobes at multiple frequencies, as shown in Fig. 1, and is frequency dependent and directional. BBSAN is less intense in the downstream direction than mixing noise due to refraction effects. In the sideline and upstream directions BBSAN $(\psi=90$ and $\psi=50$ deg. in Fig. 1 respectively) is dominant over the mixing noise for a large range of frequencies. The frequency of BBSAN varies with observer angle and jet operating conditions. The peak frequencies of BBSAN, which correspond to the peaks of each BBSAN lobe, were first successfully predicted by Harper-Bourne and Fisher. ${ }^{9}$

BBSAN intensity is observed to increase with increasing nozzle pressure ratio, $N P R$, which is the ratio of the jet stagnation pressure in the plenum of the nozzle to the static pressure of the ambient environment. The off-design parameter, $\beta$, was defined by Harper-Bourne and Fisher ${ }^{9}$ for convergent nozzles and can easily be extended to the general case as,

$$
\beta=\sqrt{\left|M_{j}^{2}-M_{d}^{2}\right|}
$$

where $M_{d}$ is the nozzle design Mach number, which is dependent on the ratio of the exit area to the throat area, and $M_{j}$ is the fully expanded Mach number, which is only dependent on the $N P R$ and the ratio of 
specific heats $\gamma$. Harper-Bourne and Fisher ${ }^{9}$ observed the intensity of BBSAN is proportional to the fourth power of $\beta$. It was shown this trend holds over a wide range of fully expanded Mach numbers for a wide range of convergent and convergent-divergent nozzles. Note that the $4^{\text {th }}$ power may vary slightly due to small effects of heating and sound emission angle, (see Viswanathan et al. ${ }^{17}$ ). At higher $\beta$ the relationship is no longer linear and the slope of $\beta^{4}$ versus $N P R$ drops off slightly. The relationship changes due to the formation of normal shocks. The scaling of BBSAN with increasing stagnation pressure is relatively well-understood by the community compared to other jet noise source mechanisms.

The question arises regarding how BBSAN scales with increasing temperature. Unfortunately, there is no first principles mathematical model or physical understanding of how BBSAN scales with increasing stagnation temperature. Specifically, the physical mechanism responsible for scaling of BBSAN intensity with respect to total temperature ratio, $T T R$ (the stagnation temperature in the nozzle plenum divided by the ambient static temperature) remains unknown. The peculiar behavior of the scaling of BBSAN with increasing $T T R$ can be observed experimentally as shown in Fig. 3. The trend is similar (in terms of intensity scaling) across observer angles, jet Mach numbers, and nozzle geometries. The phenomenon is summarized excellently by Viswanathan et al. ${ }^{17}$ who state, "The levels increase as the jet is first heated; however, the levels do not increase with further increase in jet temperature. The physical phenomenon responsible for this saturation of levels is not known at this time."

Tam ${ }^{18}$ developed a method for BBSAN prediction and the basic physical model is described by Tam and Tanna. ${ }^{19}$ Tam argued that the shock cell structure in the jet could be modeled, following the work of Pack, ${ }^{20}$ as a waveguide, where the waves are forced by the pressure imbalance at the jet exit and are confined by the jet shear layer. The simplest model that can be used for a jet is a vortex sheet. The large-scale turbulence in the jet shear layer is modeled as a random superposition of instability waves supported by the jet mean flow, as described by Tam and Chen. ${ }^{21}$ The predictions give good agreement with noise measurements in both the near- and far-field of the jet and certain key features of the measured spectra are captured. More recent versions of the Aircraft Noise Prediction Program (ANOPP) ${ }^{22}$ have incorporated Tam's BBSAN model. $\mathrm{Tam}^{23}$ modified the model by $\mathrm{Tam}^{18}$ to include the capability to predict BBSAN from heated jets up to a moderate off-design parameter. This was accomplished by better approximating the shock cell spacing. Also, a temperature correction factor, $T_{c f}$, was included to correct for the over-prediction at all frequencies due to increasing $T T R$. Tam used the following empirical correction factor for heated jet predictions,

$$
T_{c f}=\frac{\rho_{j}}{\rho_{\infty}}\left(1+\frac{\gamma-1}{2} M_{j}^{2}\right)^{-1}
$$

where $\rho_{j}$ is the fully expanded density, and $\rho_{\infty}$ is the ambient static density. As TTR increases while holding $M_{j}$ constant, the variation of $\rho_{j}$ decreases, resulting in $T_{c f}$ varying slowly at high $T T R$.

Viswanathan et al. ${ }^{17}$ performed a comprehensive study of BBSAN scaling with NPR and TTR. The BBSAN component of jet noise was isolated from the mixing noise component by subtracting the similarity spectrum of mixing noise from the total noise spectrum using the method of Viswanathan. ${ }^{24}$ This allowed a very detailed examination of the scaling of BBSAN relative to emission angle, $N P R$, and TTR.

Kuo et al. ${ }^{25}$ performed experiments that examined the effects of heating on BBSAN in the far-field by examining three nozzle geometries. The first was convergent and the others were convergent-divergent at $M_{d}=1.50$ and $M_{d}=1.76$. Heating of the jet flow was accomplished by simulating a heated flow with a helium-air mixture. Experimental measurements are shown to be consistent with those of heated air. For example, Doty and McLaughlin ${ }^{26}$ shown that helium-air jets and heated jets have similar physical and acoustic properties in the far-field. Kuo et al. ${ }^{25}$ examined heating effects for the $M_{d}=1.50$ nozzle at $M_{j}=$ $1.2,1.4,1.7$, and 1.9 by varying $T T R$ from 1.0 to 2.2 , which is well beyond the BBSAN intensity saturation point normally observed near $T T R=1.8$. An empirical model using a Gaussian envelope was formed that fits the peak BBSAN lobe extremely well.

Morris and Miller ${ }^{27}$ formed the first acoustic analogy for BBSAN. The governing equations were transformed into a left hand side operator of the linearized Euler equations (LEE) and right hand side equivalent sources. A model was then formed for the equivalent sources of BBSAN. The developed model predicts the scaling of BBSAN for a wide variety of fully expanded Mach numbers and temperature ratios, for cylindrical, dualstream, and rectangular nozzles, with over- and under-expanded jet plumes. To account for the slight heating effects on the predicted BBSAN relative to experimental data, Eqn. 2 was used to scale the spectral density.

Harper-Bourne and Fisher ${ }^{9}$ primarily studied cold jets and some jets heated to 1100 Kelvin; however, 
results of the heated cases were not presented. In their findings, Harper-Bourne and Fisher ${ }^{9}$ state, "... the intensity of shock noise is a function only of pressure ratio, and is independent of jet stagnation temperature and hence jet efflux velocity." This observation is in the context of a larger study and conflicts with many recent experimental observations.

Consider the original statement by Harper-Bourne and Fisher ${ }^{9}$ regarding BBSAN being independent of $T T R$. In the experiments of Viswanathan et al. ${ }^{17}$ and Kuo et al. ${ }^{25}$ there are noticeable differences in BBSAN intensity when holding NPR constant and varying TTR. These differences due to variation of $T T R$ are often unnoticeable if the jet is non-screeching (when the jet is heated) compared to a jet that is screeching (when the jet is cold), which is often the case in small laboratory experiments. This is due to the very large effect that screech tones have on BBSAN. Specifically, it is observed that discrete tones due to shock-associated noise decrease the peak frequencies of the broadband component and raise its amplitude. It is important to isolate the scaling of BBSAN intensity from the varying $N P R$ and from the effect of various screech intensities (which are a function of $N P R$ and $T T R$ ). This final point is extremely important in the context of this work.

In this paper, the acoustic analogy of Morris and Miller ${ }^{27}$ for BBSAN is examined with respect to the scaling of BBSAN with stagnation temperature. This is accomplished by examining the peak intensity at the sideline location. The scaling is compared with the experiments of Kuo et al. ${ }^{25}$ for the $M_{d}=1.50$ nozzle at over- and under-expanded conditions and for a $M_{d}=1.00$ nozzle, over a wide range of TTR from one to four. The equivalent source of the BBSAN is modeled with the use of local instead of ambient quantities. The new equivalent source combined with accurate calculations of the propagation of the sound through the jet shear layer, using an adjoint vector Green's function solver of the LEE, allows for predictions that retain the scaling with respect to NPR and allows for the saturation of BBSAN with increasing TTR. This is an extremely minor change to the source model of Morris and Miller. ${ }^{27}$ In the next section, the equations for the scaling law development are presented. Results of the steady Reynolds Averaged Navier-Stokes (RANS) solutions are shown, which inform the equivalent source. The saturation of the BBSAN from a single shock wave shear layer interaction using the newly developed model is shown for four cases.

\section{Mathematical Analysis}

\section{Acoustic Analogy using Propagation Effects}

The Euler equations are rearranged into a linear left hand side operator of the LEE and right hand side equivalent sources. The equivalent source of the continuity equation is the dilatation, and the equivalent source of the momentum equation is the unsteady force per unit volume involving velocity fluctuations of the shocks and turbulence in the jet plume. The latter is of interest for BBSAN prediction. The acoustic pressure is found from the convolution integral of the vector Green's function with the equivalent source. The spectral density is then formulated by the Fourier transform of the autocorrelation involving acoustic pressure. The full details of this approach are shown in Morris and Miller ${ }^{27}$ and results in,

$$
S(\underline{x}, \omega)=\rho_{\infty}^{2} c_{\infty}^{4} \int_{-\infty}^{\infty} \ldots \int_{-\infty}^{\infty} \sum_{n=1}^{3} \sum_{m=1}^{3} \pi_{g}^{n *}(\underline{x}, \underline{y}, \omega) \pi_{g}^{m}(\underline{x}, \underline{y}+\underline{\eta}, \omega) R_{n m}^{v}(\underline{y}, \underline{\eta}, \tau) \exp [-i \omega \tau] d \tau d \underline{\eta} d \underline{y}
$$

where $S$ is the spectral density, $\pi_{g}^{n}$ are the vector Green's function of the LEE, $R_{m n}^{v}(\underline{y}, \underline{\eta}, \tau)$ is the two-point cross-correlation of the equivalent source, $\underline{\eta}$ is a vector between two spatial locations in the jet source region, $\underline{x}$ is a vector from the nozzle exit to the observer, and $\underline{y}$ is a vector from the nozzle exit to a source in the jet.

The vector Green's function of the LEE as shown in Eqn. 3 are defined by the the solution of,

$$
\frac{D_{o} \pi_{g}^{n}}{D t}+\frac{\partial u_{g i}^{n}}{\partial x_{i}}=\delta(\underline{x}-\underline{y}) \delta(t-\tau) \delta_{0 n}
$$

and,

$$
\frac{D_{o} u_{g i}^{n}}{D t}+u_{g j} \frac{\partial \bar{u}_{i}}{\partial x_{j}}+\bar{c}^{2} \frac{\partial \pi_{g}^{n}}{\partial x_{i}}=\delta(\underline{x}-\underline{y}) \delta(t-\tau) \delta_{i n}
$$

where $D_{o}$ is the material derivative about the meanflow. The vector Green's function is periodic, 


$$
\pi_{g}^{n}(\underline{x}, \underline{y}, \omega)=\int_{-\infty}^{\infty} \pi_{g}^{n}(\underline{x}, \underline{y}, t-\tau) \exp [-i \omega(t-\tau)] d \tau
$$

and,

$$
\pi_{g}^{n}(\underline{x}, \underline{y}, t-\tau)=\frac{1}{2 \pi} \int_{-\infty}^{\infty} \pi_{g}^{n}(\underline{x}, \underline{y}, \omega) \exp [i \omega(t-\tau)] d \omega .
$$

The periodic vector Green's function of the LEE has the identity,

$$
\pi_{g}^{n *}(\underline{x}, \underline{y}, \omega)=\pi_{g}^{n}(\underline{x}, \underline{y},-\omega) .
$$

General analytic solutions of Eqns. 4 and 5 are unknown. However, numerical solutions can be found that are related to Lilley's ${ }^{28}$ equation. A strategy to find highly accurate numerical solutions of the vector Green's function is discussed in Appendix A.

$R_{n m}^{v}$ is the two-point cross-correlation of the equivalent source $f_{i}^{v} . R_{n m}^{v}$ takes the form,

$$
R_{n m}^{v}(\underline{y}, \underline{\eta}, \tau)=\overline{f_{n}^{v}(\underline{y}, t) f_{m}^{v}(\underline{y}+\underline{\eta}, t+\tau)}
$$

where $f_{i}^{v}$ is the equivalent source involving second order fluctuations of the momentum term in the governing equations, which is defined as,

$$
f_{i}^{v}=-u_{s j} \frac{\partial u_{t i}}{\partial x_{j}}-u_{t j} \frac{\partial u_{s i}}{\partial x_{j}}
$$

where $u$ are velocity fluctuations associated with the shocks, $s$, and the turbulence, $t$, and $x_{i}$ are independent spatial coordinates. In Morris and Miller ${ }^{27}$ the equivalent source is formulated based on dimensional and physical arguments as,

$$
f_{i}^{v} \simeq \frac{p_{s} u_{t}}{\rho_{\infty} c_{\infty} l}
$$

where $c$ is the speed of sound, $l$ is the integral turbulent length scale in the streamwise direction, $p_{s}$ is the pressure due to the shock waves, and $\rho$ is the density. In this work we assume that the density and streamwise velocity are local instead of ambient values,

$$
f_{i}^{v} \simeq \frac{p_{s} u_{t}}{\rho u l}
$$

A model for $\overline{f_{n}^{v}(\underline{y}, t) f_{m}^{v}(\underline{y}+\underline{\eta}, t+\tau)}$ is formed,

$$
\overline{f_{n}^{v}(\underline{y}, t) f_{m}^{v}(\underline{y}+\underline{\eta}, t+\tau)}=\frac{p_{s}(\underline{y}) p_{s}(\underline{y}+\underline{\eta})}{\rho^{2} c^{2} l^{2}} R(\underline{y}, \underline{\eta}, \tau)
$$

where $R(\underline{y}, \underline{\eta}, \tau)$ is the two-point cross-correlation of the velocity fluctuations. Assume that the time and spatial terms of $R(\underline{\eta}, \tau)$ are separable as Ribner postulated ${ }^{29}$ and model the two point cross-correlation of the fluctuating turbulent velocity as,

$$
R(\underline{y}, \underline{\eta}, \tau)=a_{m n} K(\underline{y}) \exp \left[-\tau^{2} / \tau_{s}^{2}\right] \exp \left[-\left(\xi-u_{c} \tau\right)^{2} / l^{2}\right] \exp \left[-\left(\eta^{2}+\zeta^{2}\right) / l_{\perp}^{2}\right]
$$

where $a_{m n}$ are coefficients that can be set for anisotriopic turbulence.

Substituting Eqns. 13 and 14 into Eqn. 3 and isolating the integral involving $\tau$ yields,

$$
\int_{-\infty}^{\infty} \exp [i \omega \tau] \exp \left[-\tau^{2} / \tau_{s}^{2}\right] \exp \left[-\left(\xi-u_{c} \tau\right)^{2} / l^{2}\right] d \tau
$$

Integration of expression 15 is performed analytically,

$$
\frac{\pi^{1 / 2} \exp \left[\frac{-4 \xi^{2}+4 i \tau_{s}^{2} u_{c} \xi \omega-l_{s}^{2} \tau_{s}^{2} \omega^{2}}{4\left(l_{s}^{2}+\tau_{s}^{2} u_{s}^{2}\right)}\right]}{\sqrt{1 / \tau_{s}^{2}+u_{c}^{2} / l_{s}^{2}}} .
$$

Expression 16 is used with Eqn. 3, 


$$
\begin{aligned}
& S(\underline{x}, \omega)=\rho_{\infty}^{2} c_{\infty}^{4} \int_{-\infty}^{\infty} \ldots \int_{-\infty}^{\infty} \sum_{n=1}^{3} \sum_{m=1}^{3} \pi_{g}^{n *}(\underline{x}, \underline{y}, \omega) \pi_{g}^{m}(\underline{x}, \underline{y}+\underline{\eta}, \omega) \\
& \times \frac{p_{s}(\underline{y}) p_{s}(\underline{y}+\underline{\eta})}{\rho^{2} c^{2} l^{2}} a_{m n} K(\underline{y}) \frac{\pi^{1 / 2} \exp \left[\frac{-4 \xi^{2}+4 i \tau_{s}^{2} u_{c} \xi \omega-l_{s}^{2} \tau_{s}^{2} \omega^{2}}{4\left(l_{s}^{2}+\tau_{s}^{2} u_{s}^{2}\right)}\right]}{\sqrt{1 / \tau_{s}^{2}+u_{c}^{2} / l_{s}^{2}}} \exp \left[-\left(\eta^{2}+\zeta^{2}\right) / l_{\perp}^{2}\right] d \underline{\eta} d \underline{y} .
\end{aligned}
$$

Over the distance where the spatial correlation is significant we assume that,

$$
\pi_{g}^{m}(\underline{x}, \underline{y}+\underline{\eta}, \omega)=\pi_{g}^{m}(\underline{x}, \underline{y}, \omega) \exp \left[i \frac{\omega}{c_{\infty}} \underline{\underline{x}} \cdot \underline{\eta}\right]
$$

as argued by Tam and Auriault. ${ }^{30}$ We now examine the term $p_{s}(\underline{y}+\underline{\eta})$ shown in Eq. 17. Morris and Miller ${ }^{27}$ noted that the variation of the Fourier transform of the shock pressure is,

$$
p_{s}\left(k_{1}, y_{2}, y_{3}\right)=\int_{-\infty}^{\infty} p_{s}(\underline{y}) \exp \left[i k_{1} y_{1}\right] d y_{1}
$$

where $k$ is the spatial wavenumber. It is observed that the variation of $p_{s}(k, \eta, \zeta)$ changes little across the jet core and shear layer where the BBSAN source is located and is certainly a valid approximation as long as the variation is small within regions of high correlation of shock pressure. Likewise, the same argument applies in the untransformed domain in conjunction with the observation that the spreading rate of jet is small and that the shock cell interactions generally occur at the same radius. With these assumptions it is argued,

$$
p_{s}(\underline{y}+\underline{\eta}) \simeq p_{s}(\underline{y}+\xi) .
$$

Morris and Miller ${ }^{27}$ used Eqn. 19 to transform the shock pressure to the wavenumber domain. This results in higher accuracy predictions relative to the use of Eqn. 20, and explicitly allows the shock cell structure to be linked to the peak and sub-peaks of the BBSAN. Here, we choose to use Eqn. 20 as it makes the analysis simpler regarding the scaling terms. In addition, the scaling remains equivalent to the previous acoustic analogy model.

Substituting Eqns. 18 and 20 into Eqn. 17 and isolating the terms of $\eta$ and $\zeta$ yields an expression of the integrals involving $\eta$ and $\zeta$,

$$
\int_{-\infty}^{\infty} \int_{\infty}^{\infty} \exp \left[\frac{-i \omega x_{2} \eta}{c_{\infty} x}\right] \exp \left[\frac{-i \omega x_{3} \zeta}{c_{\infty} x}\right] \exp \left[\frac{-\left(\eta^{2}+\zeta^{2}\right)^{2}}{l_{\perp}^{2}}\right] d \eta d \zeta .
$$

The integrals are evaluated analytically,

$$
\pi l_{\perp}^{2} \exp \left[\frac{-l_{\perp}^{2}\left(x_{2}^{2}+x_{3}^{2}\right) \omega^{2}}{4 c_{\infty}^{2} x^{2}}\right] .
$$

Expression 22 is now used to simplify the expression for the spectral density,

$$
\begin{array}{r}
S(\underline{x}, \omega)=\rho_{\infty}^{2} c_{\infty}^{4} \int_{-\infty}^{\infty} \int_{-\infty}^{\infty} \int_{-\infty}^{\infty} \sum_{n=1}^{3} \sum_{m=1}^{3} \pi_{g}^{n *}(\underline{x}, \underline{y}, \omega) \pi_{g}^{m}(\underline{x}, \underline{y}, \omega) \int_{-\infty}^{\infty} \\
\times \exp \left[\frac{-i \omega x_{1} \xi}{c_{\infty} x}\right] \frac{p_{s}(\underline{y}) p_{s}(\underline{y}+\xi)}{\rho^{2} c^{2} l^{2}} a_{m n} K(\underline{y}) \frac{\pi^{1 / 2} \exp \left[\frac{-4 \xi^{2}+4 i \tau_{s}^{2} u_{c} \xi \omega-l_{s}^{2} \tau_{s}^{2} \omega^{2}}{4\left(l_{s}^{2}+\tau_{s}^{2} u_{s}^{2}\right)}\right]}{\sqrt{1 / \tau_{s}^{2}+u_{c}^{2} / l_{s}^{2}}} \\
\times \pi l_{\perp}^{2} \exp \left[\frac{-l_{\perp}^{2}\left(x_{2}^{2}+x_{3}^{2}\right) \omega^{2}}{4 c_{\infty}^{2} x^{2}}\right] d \xi d \underline{y} .
\end{array}
$$

We now have an equivalent three dimensional model that does not require the use of the wavenumber spectrum, $\tilde{p}_{s}$. Let us now simplify the model and only examine the BBSAN source contribution from single shock locations $(\xi=0)$. Note this assumption discounts the contribution from this source location with others and other source locations with themselves, but they should all scale the same. Justification of this assumption is discussed in the following section. This operation yields, 


$$
\begin{aligned}
S_{11}(\underline{x}, \omega)= & \rho_{\infty}^{2} c_{\infty}^{4} \int_{-\infty}^{\infty} \int_{-\infty}^{\infty} \int_{-\infty}^{\infty} \sum_{n=1}^{3} \sum_{m=1}^{3} \pi_{g}^{n *}(\underline{x}, \underline{y}, \omega) \pi_{g}^{m}(\underline{x}, \underline{y}, \omega) \frac{p_{s}(\underline{y}) p_{s}(\underline{y})}{\rho^{2} c^{2} l^{2}} \\
& \times a_{m n} K(\underline{y}) \frac{\pi^{1 / 2} \exp \left[\frac{-l_{s}^{2} \tau_{s}^{2} \omega^{2}}{4\left(l_{s}^{2}+\tau_{s}^{2} u_{s}^{2}\right)}\right]}{\sqrt{1 / \tau_{s}^{2}+u_{c}^{2} / l_{s}^{2}}} \pi l_{\perp}^{2} \exp \left[\frac{-l_{\perp}^{2}\left(x_{2}^{2}+x_{3}^{2}\right) \omega^{2}}{4 c_{\infty}^{2} x^{2}}\right] d \underline{y} .
\end{aligned}
$$

$S$ is now denoted with a subscript '11' to show that the formula is only for the contribution of the BBSAN from individual shock wave shear layer interactions $(\xi=0)$. It is well known that refraction effects are negligible for observers at the sideline location relative to sound propagation through shear layers. For simplicity of examining scaling effects of BBSAN with $T T R$ we minimize the shear layer refraction effects by restricting our examination to the sideline location. At this angle, $\pi_{g}^{1}$ and $\pi_{g}^{3}$ are negligible relative to $\pi_{g}^{2}$. Thus, $\pi_{g}^{n}$ and $\pi_{g}^{m}$ are restricted to $n=2$ and $m=2$. By restricting our model to the sideline location we obtain,

$$
\begin{array}{r}
S_{11}(R, \psi \simeq \pi / 2, \omega)=\rho_{\infty}^{2} c_{\infty}^{4} \int_{-\infty}^{\infty} \int_{-\infty}^{\infty} \int_{-\infty}^{\infty} \pi_{g}^{2 *}(\underline{x}, \underline{y}, \omega) \pi_{g}^{2}(\underline{x}, \underline{y}, \omega) \frac{p_{s}(\underline{y}) p_{s}(\underline{y})}{\rho^{2} c^{2} l^{2}} \frac{a_{m n} l_{\perp}^{2} K(\underline{y}) \pi^{1 / 2}}{\sqrt{1 / \tau_{s}^{2}+u_{c}^{2} / l_{s}^{2}}} \\
\times \exp \left[\frac{-l_{s}^{2} \tau_{s}^{2} \omega^{2}}{4\left(l_{s}^{2}+\tau_{s}^{2} u_{s}^{2}\right)}\right] \exp \left[\frac{-l_{\perp}^{2}\left(x_{2}^{2}\right) \omega^{2}}{4 c_{\infty}^{2} x^{2}}\right] d \underline{y}
\end{array}
$$

Equation 25 is a volumetric integration over the source region of BBSAN that contains the shock wave shear layer interactions. It does not contain the contribution to BBSAN from multiple shock interactions. It is possible to replace the volumetric integration with a summation of small integrations over the volumes of each BBSAN source. The scaling of BBSAN with increasing TTR, as is evident from Eq. 25, scales the same for single shock interactions and adjacent shock interactions. Thus, it is simpler to examine the TTR scaling effects by only examining a single BBSAN source. For the purposes of this study the scaling of a single BBSAN source can be considered to be a single shock wave shear layer interaction. At this point the question of whether the shock noise intensity at the peak magnitude scales with the exponential terms arises. The exponential terms of Eqn. 25 have been examined in terms of scaling and fall-off. Together the exponential terms have a small effect on the scaling relative to the other terms of Eqn. 25. The terms that control the scaling of BBSAN are,

$$
S \sim \pi^{1 / 2} \rho_{\infty}^{2} c_{\infty}^{4}\left|\pi_{g}^{2}\right|^{2}(\underline{x}, \underline{y}, \omega) \frac{K(\underline{y}) p_{s}^{2}(\underline{y}) l_{\perp}^{2}}{\rho^{2} c^{2} l^{2} \sqrt{1 / \tau_{s}^{2}+u_{c}^{2} / l_{s}^{2}}} .
$$

\section{The Scaling of BBSAN with Stagnation Temperature without Propagation Effects}

The scaling of BBSAN intensity can also be examined without the effects of refraction by the jet shear layer. If the sound propagates in an environment without gradients of density or pressure, and the mean velocity field is zero, then the propagation is controlled by the Green's function of the Helmholtz equation written in terms of the vector Green's function of the LEE. The Green's function of the Helmholtz equation can be written in terms of the vector Green's function of the LEE as (for $n$ and $m$ not equal to zero),

$$
\pi_{g}^{n}(\underline{x}, \underline{y}, \omega)=\frac{i x_{n} \omega}{4 \pi c_{\infty}^{3} x^{2}} \exp \left[\frac{i \omega x}{c_{\infty}}\right] .
$$

Equation 27 multiplied by its complex conjugate is,

$$
\pi_{g}^{n *}(\underline{x}, \underline{y}, \omega) \pi_{g}^{m}(\underline{x}, \underline{y}, \omega)=\frac{x_{n} x_{m} \omega^{2}}{16 \pi^{2} c_{\infty}^{6} x^{4}} .
$$

Equation 28 and the use of the isotropic turbulence result of Proudman ${ }^{31} a_{m n} x_{n} x_{m} / x^{2}=2 / 3$, with Eqn. 23 yields, 


$$
\begin{aligned}
& S(\underline{x}, \omega)=\frac{\rho_{\infty}^{2} \omega^{2}}{16 \pi^{2} c_{\infty}^{2} x^{2}} \int_{-\infty}^{\infty} \ldots \int_{-\infty}^{\infty} \frac{p_{s}(\underline{y}) p_{s}(\underline{y}+\xi) K(\underline{y}) \pi^{1 / 2} l_{\perp}^{2}}{\rho^{2} c^{2} l^{2} \sqrt{1 / \tau_{s}^{2}+u_{c}^{2} / l_{s}^{2}}} \\
& \times \exp \left[\frac{-i \omega x_{1} \xi}{c_{\infty} x}\right] \exp \left[\frac{-4 \xi^{2}+4 i \tau_{s}^{2} u_{c} \xi \omega-l_{s}^{2} \tau_{s}^{2} \omega^{2}}{4\left(l_{s}^{2}+\tau_{s}^{2} u_{c}^{2}\right)}\right] \exp \left[\frac{-l_{\perp}^{2}\left(x_{2}^{2}+x_{3}^{2}\right) \omega^{2}}{4 c_{\infty}^{2} x^{2}}\right] d \xi d \underline{y} .
\end{aligned}
$$

We now have obtained an equation for $S$ without the Fourier transform of the shock pressure using a Helmholtz Green's function written in terms of the vector Green's function for the LEE. As before, assuming that a single shock wave shear interaction scales as adjacent ones, restricting our observer to the sideline direction $(\psi=90 \mathrm{deg})$, we can simplify Eqn. 29 as,

$$
\begin{aligned}
S_{11}(R, \psi=\pi / 2, \omega)= & \frac{\pi^{1 / 2} \rho_{\infty}^{2} \omega^{2}}{16 \pi^{2} c_{\infty}^{2} x^{2}} \int_{-\infty}^{\infty} \ldots \int_{-\infty}^{\infty} \frac{p_{s}^{2}(\underline{y}) K(\underline{y}) l_{\perp}^{2}}{\rho^{2} c^{2} l^{2} \sqrt{1 / \tau_{s}^{2}+u_{c}^{2} / l_{s}^{2}}} \\
& \quad \times \exp \left[\frac{-l_{s}^{2} \tau_{s}^{2} \omega^{2}}{4\left(l_{s}^{2}+\tau_{s}^{2} u_{c}^{2}\right)}\right] \exp \left[\frac{-l_{\perp}^{2} x_{2}^{2} \omega^{2}}{4 c_{\infty}^{2} x^{2}}\right] d \xi d \underline{y} .
\end{aligned}
$$

Similar assumptions are made here that correspond to the analysis that included propagation effects. These assumptions are: A single shock wave shear layer interaction is occurring, the observer is at the sideline location, a single source is interacting, and again the exponential terms have minimal effect on the magnitude of scaling of the shock noise, we simplify Eqn. 30 as,

$$
S \sim \frac{\pi^{1 / 2} \rho_{\infty}^{2} \omega^{2}}{16 \pi^{2} c_{\infty}^{2} x^{2}} \frac{K(\underline{y}) p_{s}^{2}(\underline{y}) l_{\perp}^{2}}{\rho^{2} c^{2} l^{2} \sqrt{1 / \tau_{s}^{2}+u_{c}^{2} / l_{s}^{2}}} .
$$

This equation represents BBSAN intensity scaling at the sideline angle, without the Fourier transform of the shock pressure, and the vector Green's function of the LEE based on spherical spreading.

\section{Results}

Four cases are selected to exercise the developed scaling laws that fit within the acoustic analogy framework of Morris and Miller. ${ }^{27}$ The cases represent over-expanded and under-expanded conditions over a range of Mach numbers for a convergent and convergent-divergent nozzle. The nozzles and operating conditions are shown in Table 1 and the TTR varies from 1.00 to 4.00 for each case. The first row of the table shows the conditions of the convergent nozzle and has corresponding data collected from the SHJAR experiment of Bridges and Brown. ${ }^{32}$ The remaining three rows of the table correspond to three of the four conditions performed in the experiment of Kuo et al. ${ }^{25}$

A CFD calculation is performed for each experimental condition summarized in Table 1. The developed acoustic analogy is informed by steady RANS solutions. The equivalent sources could easily be informed by a more advanced simulation that uses LES or simpler empirical models. The CFD simulations and their results are discussed next along with relevant field variables extracted at shock wave shear layer interactions, which are locations of the source of BBSAN. The scaling of the BBSAN with TTR is then presented based on the extracted values.

\section{Steady Reynolds-Averaged Navier-Stokes Solutions}

Wind-US (see Nelson for details ${ }^{33}$ ) was developed by the NPARC Alliance, a partnership between NASA Glenn Research Center and the U. S. Air Force Arnold Engineering Development Center, with additional contributors. A large number of validation cases for a broad range of flow-regimes have been made with the Wind-US solver by the NPARC Alliance. Wind-US can solve the Euler or Navier-Stokes equations in conjunction with many different turbulence models on structured or unstructured multi-block domains. The Wind-US CFD solver is used to calculate the steady RANS solutions of the cases shown in Table 1. Calculations are performed from $T T R=1.00$ to 4.00 in increments of 0.1 for $T T R=1.00$ to 2.50 and increments of 0.25 from $T T R=2.50$ to 4.00 . The independent variables have been normalized by the jet diameter, $D=0.0508 \mathrm{~m}$ for the SHJAR nozzle and by $D=0.0127 \mathrm{~m}$ for the Kuo et al. ${ }^{25}$ nozzle. All

simulations are performed using the axisymmetric form of the RANS equations and are closed by the Menter ${ }^{34}$ Shear Stress Transport (SST) turbulence model. Details of these types of calculations and experimental 
validation of the flow-fields are presented by Miller and Veltin. ${ }^{35}$ Some details of the CFD methodology and results are presented next. Their accuracy is important in the context of correctly informing the developed acoustic analogy and scaling laws.

Two computational domains are used for this study. The first is for the convergent nozzle, $M_{d}=1.0$, $D=0.0508 \mathrm{~m}$, and the second is for the convergent-divergent nozzle, $M_{d}=1.5, D=0.0127 \mathrm{~m}$. Both computational domains extend $75 \mathrm{D}$ from the nozzle exit in the downstream direction, $50 \mathrm{D}$ from the nozzle exit in the radial direction, and $5 D$ upstream from the nozzle inlet. Initial iterations are performed using a constant Courant-Friedrichs-Lewy (CFL) number of 0.50. Then, explicit Euler time marching using a constant time step is used until the $L^{2}$ residual remains constant and a visual inspection of the solution appears steady. The $L^{2}$ residual is a global measure of the residual error of the governing equations of the flowfield. Grid independence studies are performed by using every other grid point in the computational domain in both the streamwise and radial directions. No difference in the steady solution between the sequenced (every other grid point) and unsequenced domain is apparent in any of the solutions. The turbulent boundary layer at the nozzle exit is fully resolved by setting the first grid point in the viscous sublayer region using $y^{+}=1$, where $y^{+}$is the coordinate of the viscous wall layer using average conditions. ${ }^{36}$

Boundary conditions for the simulations consist of a downstream outflow that allows the flow to exit the computational domain. This condition assumes that the outflow is subsonic and the free-field pressure is set at the ambient pressure. Free-stream boundary conditions are specified in the free-field where a small uniform flow of $M_{\infty}=0.001$ is assumed for stability and the total ambient pressure is equal to the downstream static pressure. A standard axisymmetric boundary condition, which is essentially an inviscid wall, lies on the centerline at $r / D=0$. Adiabatic no-slip wall boundary conditions are used on the nozzle surface. Finally, the upstream boundary condition for the nozzle inlet specifies the total pressure and total temperature of the jet with an initial inlet $M=0.15$ normal to the inlet plane. The inlet $M$ may fluctuate and the total pressure and total temperature are held constant. Full details of the computational grids and boundary conditions used in this simulation can be found in Miller et al. ${ }^{35}$ The numerical implementation and mathematical development are shown in Nelson. ${ }^{33}$ These boundary conditions are sufficient for a steady solution to converge when using an ideal gas.

By examining Table 1, it can be shown that only four NPR are required and only four unique values of the off-design parameter, $\beta$, result. Changing $T T R$ while holding the NPR constant results in nearly identical shock-cell structures. The shock pressures of the four families of jets are shown in Fig. 4, which is the difference between the static pressure in the jet plume and the ambient static pressure. Shock pressure remains relatively constant for constant $N P R$ with varying $T T R$. Contours of shock pressure are shown and the axes are normalized by the jet diameter. CFD solutions are mirrored about the $x$-axis for illustration purposes. Since the steady jet flow-fields are axisymmetric, only a slice of the flow-field is used for the computational domain. The jet conditions in Fig. 4 are a) $M_{d}=1.50, M_{j}=1.20, T T R=1.00, D=0.0127$ $\mathrm{m}$, b) $M_{d}=1.00, M_{j}=1.50, T T R=1.00, D=0.0508 \mathrm{~m}$, c) $M_{d}=1.50, M_{j}=1.70, T T R=1.00$, $D=0.0127 \mathrm{~m}$, and d) $M_{d}=1.50, M_{j}=1.90, D=0.0127 \mathrm{~m}, T T R=1.00$. As the TTR varies the shock cell position remains almost constant. As the fully expanded Mach number changes between the four cases, it is evident how great the changes in the oblique shock wave angles are. The circles in parts a) through d) represent the first location where a conical oblique shock wave interacts with the jet shear layer. Subsequent shock wave shear layer interactions can be observed further downstream. The shock cell shear layer interactions represent the positions where BBSAN sources are located. At each circled location, the field variables are extracted as a function of $T T R$ for each $M_{j}$ using the steady RANS solutions. These flow-field values are functions of $T T R$ and are the expressions of the developed scaling arguments shown in the previous section.

It is informative to examine the variation of the static temperature field with the variation of $T T R$ while holding $M_{j}$ constant. For this task the third case in Table 1 is selected where $M_{d}=1.50, M_{j}=1.70$, and $D=0.0127 \mathrm{~m}$. Contours of static temperature are shown in Fig. 5 and vary from a) $T T R=1.00$, b) $T T R=1.30$, c) $T T R=1.50$, d) $T T R=1.70$, e) $T T R=1.90$, f) $T T R=2.10$, g) $T T R=2.30$, h) $T T R=2.50$, i) $T T R=3.00$, and j) $T T R=3.50$. Observe that the location of the first oblique shock wave interaction with the shear layer remains constant irregardless of the jet TTR. For low values of $T T R$, the static temperature of the jet is lower than the ambient. As $T T R$ is raised it is eventually equal to the ambient, which is the isothermal jet condition (approximately $T T R=1.70$ for this $M_{j}$ ). As the jet $T T R$ is increased higher than the isothermal jet $T T R$, the static temperature rises above the ambient. Note the cases where $T T R=1.00$ and $T T R=3.50$ have very large contrast relative to values of $T T R$ near the isothermal 
jet condition. Refraction effects are controlled more so by density gradients and velocity gradients at the sideline angle than by temperature gradients (which are related through the gas law). For the isothermal jet the density gradients are relatively small. At $T T R$ values far from the isothermal condition, the temperature and density gradients are stronger and refraction effects should be greater.

To illustrate the relative source strength and location of the BBSAN sources, a numerical investigation is performed with a $M_{d}=1.00, M_{j}=1.50, T T R=1.00, D=0.0254 \mathrm{~m}$ jet. A steady RANS solution of this flow-field is shown in Fig. 6. Part a) shows contours of shock pressure and part b) shows contours of turbulent kinetic energy, which is predominately contained within the jet shear layer before the end of the potential core. Note the unique variation of the spreading of $K$, which is due to the turning of the flow by Prandtl-Meyer expansion waves and oblique shock waves balanced by the spreading of the jet. Part c) shows contours of the integrand of the model of Morris and Miller $^{27}$ evaluated in the jet for $\psi=90$ degrees and $R / D=100$. These contours represent the relative strength of BBSAN at the peak BBSAN frequency. At this source location the high values of $p_{s}$ and $K$ can be observed in part a) and b) respectively and indeed, as theory suggests, correspond to shock wave shear layer interactions.

The quantities shown in the developed scaling equation have been extracted from the steady RANS solutions conducted at each jet condition and at locations where the oblique shock waves interact with the jet shear layer. These locations are shown by circles in Fig. 4. Coordinates of the locations are summarized in Table 2. These locations are selected by carefully examining the RANS solution contour plots of static pressure. As shown previously, the integrand of the models of Morris and Miller ${ }^{27}$ illustrate that these locations are also those that correspond to a large contribution of the BBSAN. These locations are illustrated in contour plots of the integrand as shown in Fig. 6.

\section{Variation of Field Variables at the BBSAN Source Location}

The arguments of the developed acoustic analogy and scaling terms are extracted at the first shock wave shear layer interaction of each jet family studied. This is accomplished by a small software program that iteratively examines the steady RANS solution files, extracts the values, and writes them to new files. Extracted values of quantities that control the scaling of BBSAN from the first shock wave shear layer interaction are shown in Figs. 7(a) through 12(b). The variation of these quantities with TTR are well known but quickly reviewed. Figure 7(a) and 7(b) show the static density and streamwise velocity component as a function of increasing $T T R$ for the various $M_{j}$ studied. Density falls as the inverse square of $T T R$ and the streamwise velocity component increases almost at a linear rate. Together, these quantities at the same $M_{j}$ and $T T R$ yield the ensemble average of the streamwise momentum at the BBSAN source.

Information regarding the turbulence statistics can be directly extracted from the steady RANS solutions. These include the turbulent kinetic energy and dissipation as shown in Figs. 8(a) and 8(b) respectively. As expected, both quantities are highly dependent on the TTR.

The integral length scales in the streamwise and cross-stream direction can be estimated based on a simple dimensional analysis of $K$ and $\epsilon$. Using the extracted values of $K$ and $\epsilon$ in Figs. 8(a) and 8(b), the integral streamwise and cross-stream length scales are shown in Figs. 9(a) and 9(b) respectively. The coefficients used to find $l$ and $l_{\perp}$ are $c_{l}=3.50$ and $c_{\perp}=0.30$, as found by Morris and Miller. ${ }^{27} c_{\perp}$ is chosen solely on experimental observation of the cross-stream integral length scale being approximately one-third of the streamwise integral length scale.

Figure 10(a) shows the static temperature at the first shock wave shear layer interaction for the jet families under investigation. This quantity was derived from the total energy, density, and velocities of the steady RANS solution. It has a slightly decreasing slope with increasing TTR. The local speed of sound at the same locations are shown in Fig. 10(b). Local speed of sound is calculated based on the static temperature in Fig. 10(a) and the relation $c=\sqrt{\gamma R T}$. The nonlinear factor of the speed of sound with respect to increasing $T T R$ is due to the exponent of the ideal gas law.

The Mach number as a function of TTR at the first oblique shock wave shear layer interaction is shown in Fig. 11(a). It is calculated using the corresponding velocity magnitude divided by the time averaged speed of sound shown in Fig. 10(b). It is clear that there is only a small variation of Mach number as TTR changes at the location of BBSAN sources, especially at higher TTR. The integral time scale is shown as a function of TTR in Fig. 11(b). Like the streamwise and cross-stream length scales shown in Figs. 9(a) and 9(b), it is calculated from $K$ and $\epsilon$ at the BBSAN source location.

The shock pressure is the difference between the static pressure and the ambient static pressure. It represents the pressure due to the shock cell structure within the jet plume. The BBSAN intensity is 
proportional to the shock pressure which is most affected by the off-design parameter. Shock pressure as a function of $T T R$ is shown in Fig. 12(a). Except for the family of solutions at $M_{j}=1.9$, the shock pressure is shown to be independent of TTR. The $M_{j}=1.9$ family of solutions contains some variation, but this is attributed to slight changes in the position of the oblique shock wave with increasing $T T R$, as the position which the data is extracted remains constant. A final comparison is shown for the second component of the vector Green's function pressure term of the LEE in Fig. 12(b). This factor of the Green's function component is chosen because of the form in which it appears in the scaling model. Other components are neglected. $n=1$ is neglected as it is negligible relative to $n=2$ at the sideline observer location. $n=3$ is neglected as the integration of an axisymmetric jet about the azimuthal angle $\phi$ negates the contribution from this component at the sideline observer location. At the sideline location almost no refraction effects occur except for damping or amplification of intensity due to density gradients. The density gradients are related to the TTR. It is evident from Fig. 12(b) that the refraction effects on the BBSAN at the location of the source causes the intensity to lower with $T T R$. For sideline observer locations, Eqn. 33 in Appendix A reduces to the form of $f_{r r}+\left(1 / r-\rho_{r} / r\right) f_{r}-m^{2} f / r^{2}=0$. Variation of this differential equation depends on $\partial \rho / \partial r$ at the shock wave shear layer location with varying $T T R$. A smooth variation of $\left|\pi_{g}^{2}\right|^{2}$ should be expected and is observed from the calculation.

The arguments of the scaling for BBSAN intensity have been isolated and in the next section will be evaluated to observe saturation at the sideline location from a single source.

\section{Scaling of Broadband Shock-Associated Noise with Temperature}

The mathematical models developed in the previous section are implemented in the computer program RANS Integration for Shock Noise (RISN). RISN is a collection of jet noise prediction models that are based on various acoustic analogies and is being developed at NASA Langley Research Center (LaRC). The acoustic analogies produce total noise predictions based on a summation of jet noise components from axisymmetric or three dimensional jet flow-fields that are heated, off-design, and compressible. Acoustic analogies are developed for each noise source (eg: BBSAN, fine- and large-scale mixing noise, screech, etc.) and the sources and propagation are treated separately. The predictions of this section are produced with the RISN code.

A comparison of the predicted source scaling of the BBSAN with experiment is shown in Fig. 13 for the convergent nozzle, $M_{j}=1.50$, and varying $T T R$. The predicted contribution from the first shock wave shear layer interaction (which does not include adjacent shock-shock contributions) by Eqn. 26 is shown as a black line with round circles. The source spectrum is referenced to the $y$-axis scale on the right side of the figure. The data from the SHJAR experiment is shown as red squares and is referenced to the $y$-axis on the left side of the figure. Even though the $y$-axis on the left and right have different minimum and maximum values, the scale and units are the same. This point must be stressed and is reinforced on the figure by the denotion that one decibel changes are shown in the scale and imply that the relative scaling of the experiment and the source are the same. Recall from the mathematical analysis that single shock and adjacent shock wave shear layer interactions scale the same. Summation of all the sources yields a total prediction of BBSAN that agrees with experiment. The extraction and evaluation of the scaling law has been performed based on the location shown in Fig. 4 part b) at the circled location. The prediction using only spherical spreading, the same source model (which is almost equivalent to that of Morris and Miller ${ }^{27}$ ), and no effect of the jet shear layer is shown by a dashed line with triangles. Note that the factor $T_{c f}$ has been neglected in these predictions. The inclusion of $T_{c f}$ yields saturation of the prediction of Morris and Miller ${ }^{27}$ with increasing $T T R$. It is clear that with increasing $T T R$ the prediction of Eqn. 26 initially increases linearly with $T T R$ and eventually saturates.

The following comparisons are performed with the experiment of Kuo et al. ${ }^{25}$ who used a $M_{d}=1.50$, $D=0.0127 \mathrm{~m}$ nozzle, and varied the TTR. Figure 14 shows comparisons at $R / D=100$ and $\psi=90$ degrees with $M_{j}=1.20$. Experimental values represent the maximum BBSAN at the sideline location of the jet. This particular jet condition throughout its temperature range produced no screech tones. The agreement between the scaling of the prediction and the experiment is excellent. The scaling of the source shows a clear increase from $T T R=1.00$ and saturates at the same rate as the experiment.

For the next comparison, the same nozzle and observer location is retained but the jet operates at $M_{j}=1.70$. Comparisons between the predicted peak BBSAN and Kuo et al. ${ }^{25}$ are shown in Fig. 15. Unlike the previous case, these jet conditions throughout the low temperature range exhibit very strong screech tones. The over-pressure of the screech is marked by each data point in the figure. The over-pressure of the 
screech, $S_{o}$, is a measure of the maximum screech amplitude minus the broadband level at the fundamental screech frequency. Note that the screech frequency is often lower then the maximum BBSAN frequency. To illustrate this point, reexamine Fig. 1 at the sideline location, where the fundamental screech tone frequency is lower than the peak BBSAN frequencies. The tone labeled screech is the fundamental screech tone and its overpressure is approximately $12 \mathrm{~dB}$. In Fig. 15 the maximum BBSAN has corresponding values of $S_{o}$ that change with $T T R$. At low temperatures the screech over-pressure is large and as the TTR increases the screech over-pressure approaches zero. At the sideline location screech has a large effect on BBSAN. In Fig. 13 where $S_{o}$ is relatively constant and non-zero and Fig. 14 where there is no screech, the agreement between prediction and experiment is very good. If the screech over-pressure is constant through the range of $T T R$ or preferably, zero, as it is typically in full-scale engines unlike small scale nozzles, then the effects of screech on the BBSAN are relatively the same and the scaling of BBSAN is accurately captured. In Fig. 15, one may observe the correct trend of BBSAN saturation starting at TTR $=1.4$. The BBSAN is amplified a large amount due to the large screech amplitudes present at low temperatures.

A final comparison is made between the prediction of the scaling using Eqn. 26 and the experiment of Kuo et al. ${ }^{25}$ in Fig. 16. The fully expanded Mach number is increased to 1.90 and all other conditions are retained. Once again, the screech over-pressure is labeled at each data point. $S_{o}$ is relative small past $T T R=1.4$. The agreement between the source scaling prediction and experiment of Kuo et al. ${ }^{25}$ at moderate to higher temperatures is excellent. At low temperatures the screech tones have disrupted the trend due to reasons previously described, and the correct scaling of BBSAN is not captured. Recall that at the sideline location the addition of discrete tones lowers the peak BBSAN frequency and raises the peak BBSAN magnitude. This is exactly what is observed in the experimental data. If screech was not present within the experiments then the trends at low TTR for Figs. 15 and 16 will yield a lowered BBSAN amplitude, and eventual saturation as seen in the experiments of Figs. 13 and 14 will occur.

Based on the previous four comparisons, it is evident that the inclusion of using local properties for the streamwise velocity component and density, instead of ambient quantities, and the combination of the vector Green's function that is amplified by the shear layer, yields a model that is consistent with experiment. This is accomplished by only changing the values of $\rho_{\infty}$ and $u_{\infty}$ of the model of Miller and Morris ${ }^{37}$ to $\rho$ and $u$. This captures the correct scaling of BBSAN with both $N P R$ and TTR.

Unheated conditions are difficult to predict due to the rapid change of BBSAN intensity. Not only are the predictions very difficult to conduct both mathematically and in terms of implementation but the experiments are very difficult to perform, especially with nozzles on the order of $10^{-2}$ meters. The experimental approaches with their associated sensitivity and difficulty cannot be understated.

Screech tones are extremely sensitive to laboratory conditions and are highly chaotic, however, all fluid dynamic phenomena are deterministic. When the screech over-pressure is very high at low TTR, the BBSAN is lowered in frequency and raised in amplitude. It is possible for an experiment to be performed for the same jet conditions as shown in this paper, but without screech and without its effect on the mixing noise or BBSAN. It is expected with great confidence that the peak BBSAN intensity levels, without the influence of the discrete tone, will compare with the developed theory.

\section{Conclusion}

BBSAN intensity saturates with increasing jet stagnation temperature. This saturation occurs due to the balance between the source term and the propagation effects. An acoustic analogy for BBSAN, which is a modification of the model of Morris and Miller ${ }^{27}$ is proposed that takes into account the scaling of both NPR and TTR accurately. This model results from a simple modification that involves using local values of $\rho$ and $u$ instead of ambient values for the source term and includes accurate shear layer refraction effects. The latter is achieved by calculating the adjoint Green's function of Lilley's equation and relating it to the vector Green's function of the LEE. This approach is highly accurate and efficient in the sideline direction. The source scaling term is contained within the developed acoustic analogy and contains the effects of the equivalent source and propagation separately. This term is extracted from the acoustic analogy and evaluated with arguments from four families of supersonic off-design jets. Evaluation involves extracting the local properties at the shock wave shear layer interaction from steady RANS solutions. These local field variables are the arguments of the source term. Comparisons of the predicted BBSAN from a single shock wave shear layer interaction compare very favorably with experimental data. The predictions, like the experiments, show eventual saturation with increasing jet stagnation temperature. Additional interactions 
can include other shock wave shear layer interactions, adjacent shock wave shear layer interactions, etc. The source considered in this work has the same scaling characteristics of the other shock wave shear layer interactions.

\section{Acknowledgements}

The author benefited greatly from discussions with Boeing/A. D. Welliver Professor Philip J. Morris of the Pennsylvania State University. The availability of experimental data from Professor Dennis K. McLaughlin of the Pennsylvania State University and from Dr. James Bridges of NASA Glenn Research Center at Lewis Field made this work possible. The author is grateful for continuous support from The National Aeronautics and Space Administration (NASA) Fundamental Aeronautics Program (FAP) Supersonics Project (SUP).

\section{Appendix A}

This mathematical analysis requires knowledge of the vector Green's function of the LEE. An adjoint approach is followed here which shortens calculation time greatly. This approach is based on the work of Tam and Auriault ${ }^{30}$ and Raizada. ${ }^{38}$ Assuming a locally parallel mean flow, $q=q(y, z)$, where $q$ represents the field-variables, the adjoint Green's function can be written,

$$
g_{a}(\underline{x}, \underline{y}, \omega)=\frac{\exp \left[-i \omega\left(x_{1} \cos \theta-R\right) / c_{\infty}\right]}{8 \pi^{2} c_{\infty}^{2} R \omega} \sum_{m=0}^{\infty} f_{m}(r) \cos (m \phi)
$$

where $m$ is an azimuthal mode number. Equation 32 is valid inside the jet $\left(r<R_{o}\right)$. There is no concern about solutions outside the jet as sources of BBSAN do not exist in that region. $f_{m}$ is found for each mode $m$ by solving the following differential equation in the region $0<r<R_{o}$,

$$
\begin{aligned}
& \frac{\partial^{2} f_{m}}{\partial r^{2}}+\left[\frac{-4 \cos \theta \frac{d \bar{u}}{d r} / c_{\infty}}{1-\bar{u} \cos \theta / c_{\infty}}-\frac{1}{\bar{\rho}} \frac{d \bar{\rho}}{d r}+\frac{1}{r}\right] \frac{\partial f_{m}}{\partial r} \\
& +\left[\frac{\omega^{2}\left(1-\frac{u \cos \theta}{c_{\infty}}\right)}{\gamma p / \rho}+\frac{3\left(\frac{1}{\rho} \frac{\partial \rho}{\partial r} \frac{\partial u}{\partial r}-\frac{\partial^{2} u}{\partial r^{2}}\right) \frac{\cos \theta}{c_{\infty}}}{1-u \cos \theta / c_{\infty}}-\frac{m^{2}}{r^{2}}-\frac{\omega^{2} \cos ^{2} \theta}{c_{\infty}^{2}}\right] f_{m}=0
\end{aligned}
$$

As shown by Tam and Auriault, ${ }^{30}$ the solution near the centerline $(r \simeq \epsilon)$ is $f_{m}=a_{m} r^{m}$. Using this expression, the boundary conditions at the centerline of the jet are specified, and a spatial marching problem in the radial direction can be formed at each streamwise location. The details of the numerical procedure to solve Eqn. 33 are shown in Raizada. ${ }^{38}$ For each radial mode $m, f_{m}$ needs to be scaled so that the innersolution matches the outer-solution of $g_{a}$ at $r=R_{o}$. Raizada ${ }^{38}$ showed that a scaling factor can be used for this purpose,

$$
a_{m}=\frac{(-i)^{m} \epsilon_{m} \lambda\left(J_{m}\left(\lambda R_{o}\right) H_{m}^{(1)^{\prime}}\left(\lambda R_{o}\right)-J_{m}^{\prime}\left(\lambda R_{o}\right) H_{m}^{(1)}\right)}{f_{m}\left(R_{o}\right) H_{m}^{(1)}\left(\lambda R_{o}\right) f_{m}^{\prime}\left(R_{o}\right)-H_{m}^{(1)}\left(\lambda R_{o}\right) \lambda f_{m}\left(R_{o}\right)}
$$

where $\epsilon_{m}$ is unity for $m=1$ and is two for all other values of $m, \lambda=\omega \sin \theta / c_{\infty}$, and the primes denote a derivative of the Hankel function or $f_{m}$ with respect to their arguments. The vector Green's function of the LEE is related to the Green's function of Lilley's equation by,

$$
\begin{gathered}
\pi_{g}^{0}=\omega^{2} g-2 i u \omega \frac{\partial g}{\partial y_{x}}-u \frac{\partial^{2} g}{\partial y_{x}^{2}} \\
\pi_{g}^{1}(\underline{x}, \underline{y}, \omega)=-\left(i \omega+u \frac{\partial}{\partial y_{x}}\right) \frac{\partial}{\partial y_{x}} g(\underline{x}, \underline{y}, \omega) \\
\pi_{g}^{2}(\underline{x}, \underline{y}, \omega)=-\left\{3 \frac{\partial u}{\partial y_{r}} \frac{\partial}{\partial y_{x}}+\left(i \omega+u \frac{\partial}{\partial y_{x}}\right) \frac{\partial}{\partial y_{r}}\right\} g(\underline{x}, \underline{y}, \omega) \\
\pi_{g}^{3}(\underline{x}, \underline{y}, \omega)=-\left(i \omega+u \frac{\partial}{\partial y_{x}}\right) \frac{1}{y_{r}} \frac{\partial}{y_{\theta}} g(\underline{x}, \underline{y}, \omega)
\end{gathered}
$$


where $\underline{y}=\left(y_{r}, y_{\theta}, y_{x}\right)$ denotes the source position in cylindrical polar coordinates. The reciprocal relation of $g=\bar{g}_{a}$ holds true for this formulation of the adjoint Green's function. Equations 35 through 38 require various terms involving derivatives for the Green's function of Lilley's equation. These expressions are found analytically. The various derivatives of $g$ used in Eqns. 35 through 38 with respect to the various spatial directions are,

$$
\begin{gathered}
\frac{\partial g}{\partial x}=\frac{-i \cos \theta}{8 \pi^{2} c_{\infty}^{3} R} \exp \left[-i \omega(x \cos \theta-R) / c_{\infty}\right] \sum_{m=0}^{\infty} f_{m}(r) \cos (m \phi) \\
\frac{\partial^{2} g}{\partial x^{2}}=\frac{-\omega \cos ^{2} \theta}{8 \pi^{2} c_{\infty}^{4} R} \exp \left[-i \omega(x \cos \theta-R) / c_{\infty}\right] \sum_{m=0}^{\infty} f_{m}(r) \cos (m \phi) \\
\frac{\partial g}{\partial \phi}=\frac{-m}{8 \pi^{2} \omega c_{\infty}^{2} R} \exp \left[-i \omega(x \cos \theta-R) / c_{\infty}\right] \sum_{m=0}^{\infty} f_{m}(r) \sin (m \phi) \\
\frac{\partial}{\partial x}\left(\frac{1}{r} \frac{\partial g}{\partial \phi}\right)=\frac{i m \cos \theta}{8 r \pi^{2} c_{\infty}^{3} R} \exp \left[-i \omega(x \cos \theta-R) / c_{\infty}\right] \sum_{m=0}^{\infty} f_{m}(r) \sin (m \phi)
\end{gathered}
$$

Equations 39 through 42 are used with Eqns. 35 through 38 to find the vector Green's function based on Lilley's equation at various streamwise locations. In this work only a single streamwise location is used that corresponds to the location of the first shock wave shear layer interaction.

\section{References}

${ }^{1}$ Powell, A., "Structural Fatigue Due to Jet Noise," Journal of the Acoustical Society of America, Vol. 28, No. 4, 1956, pp. $782-782$.

${ }^{2}$ Clark, C. and Stansfeld, S. A., "The Effect of Transportation Noise on Health and Cognitive Development: A Review of Recent Evidence," International Journal of Comparative Psychology, Vol. 20, No. 2, 2007, pp. 145-158.

${ }^{3}$ Tedrick, R. N. and Hixson, R. B., "Aircraft Noise and the Airport Community," AIAA International Air Transportation Conference, Montreal, Canada, AIAA Paper 83-1580, 1983.

${ }^{4}$ Viswanathan, K., "Aircraft Noise: An Overview," Proceedings of the International Conference on Aerospace Science and Technology, 26-28, June, 2008.

${ }^{5}$ Bowes, W. C., Rumpf, D., Bowler, D. R., Carnes, R. S., Fratarangelo, P. A., Huff, D. L., Moin, P., and Voorhees, W. J., "Jet Engine Noise Reduction," Naval Research Advisory Committee, April 2009, pp. 1-69.

${ }^{6}$ Crocker, M. J., "The Vibroacoustic Environment of Spacecraft During Launch and Flight," Sound and Vibration, Vol. 36, No. 2, 2002, pp. 5 .

${ }^{7}$ Arenas, J. P. and Margasahayam, R. N., "Noise and Vibration of Spacecraft Structures," Revista Chilena de Ingenieria, Vol. 14, No. 3, September 2006, pp. 251-264.

${ }^{8}$ Powell, A., "On the Noise Emanating from a Two-Dimensional Jet above the Critical Pressure," Aeronautical Quarterly, Vol. 4, 1953, pp. 103-122.

${ }^{9}$ Harper-Bourne, M. and Fisher, M. J., "The Noise from Shock-Waves in Supersonic Jets," AGARD Conference Proceedings, 1973.

${ }^{10}$ Ffowcs-Williams, J. E., "Hydrodynamic Noise," Annual Review of Fluid Mechanics, Vol. 1, 1969, pp. $197-222$.

${ }^{11}$ Ffowcs-Williams, J. E., "Aeroacoustics," Annual Review of Fluid Mechanics, Vol. 9, 1977, pp. 447-68.

${ }^{12}$ Goldstein, M. E., "Aeroacoustics of Turbulent Shear Flows," Annual Review of Fluid Mechanics, Vol. 16, 1984, pp. 263285.

${ }^{13}$ Tam, C. K. W., "Supersonic Jet Noise," Annual Review of Fluid Mechanics, Vol. 27, 1995, pp. 17-43.

${ }^{14}$ Zaman, K. B. M. Q., Bencic, T., Clem, M., and Fagan, A., "Shock-Induced Boundary Layer Separation in C-D Nozzles and Its Impact on Jet Noise," 49th AIAA Aerospace Sciences Meeting, Orlando, Florida, AIAA Paper 2011-1031, 2011.

${ }^{15}$ Tam, C. K. W., "Mach Wave Radiation from High-Speed Jets," AIAA Journal, Vol. 47, No. 10, 1999, pp. $2440-2448$.

${ }^{16}$ Raman, G., "Supersonic Jet Screech: Half-Century from Powell to the Present," Journal of Sound and Vibration, Vol. 225, No. 3, 1999, pp. 543-571.

${ }^{17}$ Viswanathan, K., Alkislar, M. B., and Czech, M. J., "Characteristics of the Shock Noise Component of Jet Noise," AIAA Journal, Vol. 48, No. 1, 2010, pp. 25-46.

${ }^{18}$ Tam, C. K. W., "Stochastic Model Theory of Broadband Shock-Associated Noise from Supersonic Jets," Journal of Sound and Vibration, Vol. 116, No. 2, 1987, pp. 265-302.

${ }^{19}$ Tam, C. K. W. and Tanna, H. K., "Shock-Associated Noise of Supersonic Jets from Convergent-Divergent Nozzles," Journal of Sound and Vibration, Vol. 81, No. 3, 1982, pp. 337-358.

${ }^{20}$ Pack, D. C., "A Note on Prandtl's Formula for the Wavelength of a Supersonic Gas Jet," Quarterly Journal of Applied Mathematics and Mechanics, Vol. 3, 1950, pp. 173-181.

${ }^{21}$ Tam, C. K. W. and Chen, K. C., "A Statistical Model of Turbulence in Two-Dimensional Mixing Layers," Journal of Fluid Mechanics, Vol. 92, 1979, pp. 303-326. 
${ }^{22}$ Zorumski, W. E., "Aircraft Noise Prediction Program. Theoretical Manual. Parts 1 and 2," NASA TM-83199, 1982.

${ }^{23}$ Tam, C. K. W., "Broadband Shock-Associated Noise of Moderately Imperfectly-Expanded Supersonic Jets," Journal of Sound and Vibration, Vol. 140, No. 1, 1990, pp. 55-71.

${ }^{24}$ Viswanathan, K., "Scaling Laws and a Method for Identifying Components of Jet Noise," AIAA Journal, Vol. 44, No. 10, 2006, pp. 2274-2285.

${ }^{25}$ Kuo, C., McLaughlin, D. K., and Morris, P. J., "Effects of Supersonic Jet Conditions on Broadband Shock-Associated Noise," 49th AIAA Aerospace Sciences Meeting, AIAA Paper 2011-1032, 2011.

${ }^{26}$ Doty, M. J. and McLaughlin, D., "Acoustic and Mean Flow Measurements of High-Speed Helium-Air Mixture Jets," International Journal of Aeroacoustics, Vol. 2, No. 2, 2003, pp. 293-334.

${ }^{27}$ Morris, P. J. and Miller, S. A. E., "Prediction of Broadband Shock-Associated Noise Using Reynolds-Averaged NavierStokes Computational Fluid Dynamics," AIAA Journal, Vol. 48, No. 12, 2010, pp. 2931-2961.

${ }^{28}$ Lilley, G. M., "On the Noise from Jets," AGARD Conference Proceedings In Noise Mechanisms, Vol. 13, 1974, pp. 1-11.

${ }^{29}$ Ribner, H. S., "The Generation of Sound by Turbulent Jets," Advances in Applied Mechanics, Vol. 8, 1964, pp. 103-182.

${ }^{30}$ Tam, C. K. W. and Auriault, L., "Mean Flow Refraction Effects on Sound Radiated from Localized Sources in a Jet," Journal of Fluid Mechanics, Vol. 370, 1998, pp. 149-174.

${ }^{31}$ Proudman, J., "The Generation of Noise by Isotropic Turbulence," Proc. Roy. Soc. A., Vol. 214, No. 1116, 1952, pp. 119132 .

${ }^{32}$ Bridges, J. and Brown, C. A., "Validation of the Small Hot Jet Acoustic Rig for Aeroacoustic Research," 11th AIAA/CEAS Aeroacoustics Conference, May 23-25, AIAA Paper 2005-2846, 2005.

${ }^{33}$ Nelson, C., "An Overview of the NPARC Alliance's Wind-US Flow Solver," 48th AIAA Aerospace Sciences Meeting, AIAA Paper 2010-27, 2010.

${ }^{34}$ Menter, F. R., "Two-Equation Eddy-Viscosity Turbulence Models for Engineering Applications," AIAA Journal, Vol. 32, No. 8, 1994, pp. 1598-1605.

${ }^{35}$ Miller, S. A. E. and Veltin, J., "Assessment of Computational Fluid Dynamics for Supersonic Shock Containing Jets," AIAA Journal, Vol. 47, No. 11, 2009, pp. 2738-2746.

${ }^{36}$ Schlicting, H., Boundary-Layer Theory, Springer-Verlag, Berlin, 8th ed., 2000.

${ }^{37}$ Miller, S. A. E. and Veltin, J., "Experimental and Numerical Investigation of Flow Properties of Supersonic Helium-Air Jets," AIAA Journal, Vol. 49, No. 1, 2011, pp. 235-246.

${ }^{38}$ Raizada, N., "Numerical Prediction of Noise from High Speed Subsonic Jets using an Acoustic Analogy," Ph.D. Dissertation, The Pennsylvania State University, December 2005.

\section{Tables}

Table 1. Cases and operating conditions of the jet flows. For each row a simulation is performed at TTR 1.00 to 2.50 at increments of 0.10 and from $T T R 2.50$ to 4.00 in increments of 0.50 . In total 80 steady RANS solutions are found.

\begin{tabular}{cccc}
$M_{d}$ & $D(\mathrm{~m})$ & $M_{j}$ & $N P R$ \\
\hline 1.00 & 0.0508 & 1.50 & 2.42 \\
1.50 & 0.0127 & 1.20 & 3.67 \\
1.50 & 0.0127 & 1.70 & 4.94 \\
1.50 & 0.0127 & 1.90 & 6.70
\end{tabular}

Table 2. Coordinates of the extracted scaling arguments from the steady RANS solutions.

\begin{tabular}{ccc}
$M_{j}$ & $x(\mathrm{~m})$ & $r(\mathrm{~m})$ \\
\hline 1.20 & 0.01205 & 0.00544 \\
1.50 & 0.01938 & 0.00557 \\
1.70 & 0.02304 & 0.00594 \\
1.90 & 0.03029 & 0.00535
\end{tabular}




\section{Figures}
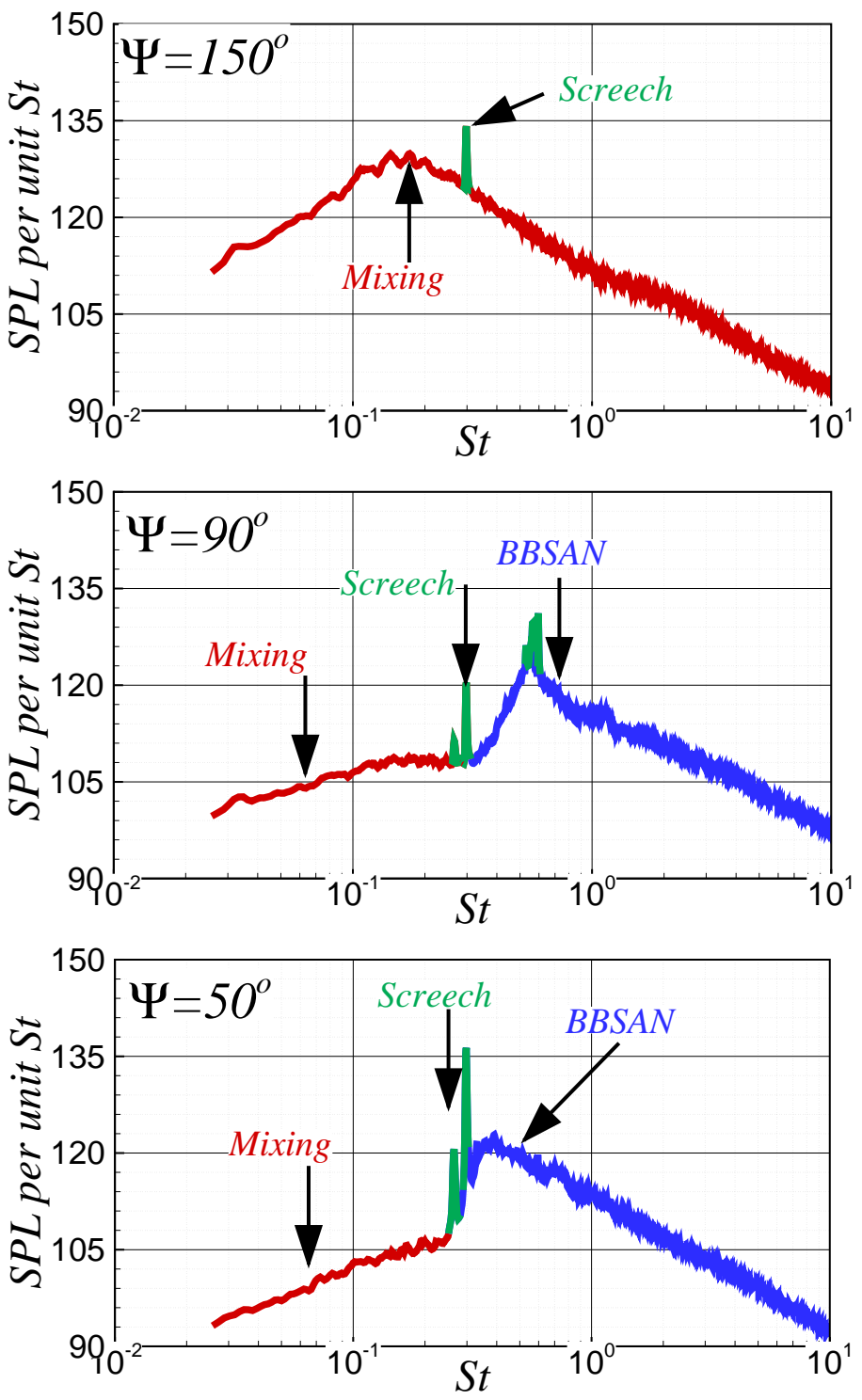

Figure 1. Sound pressure level per unit Strouhal number resulting from a $M_{d}=1.00, M_{j}=1.50$, and $T T R=1.00$ jet. The red lines represent the noise that is dominated by mixing noise, blue lines represent noise that is dominated by BBSAN, and green lines represent noise that is dominated by discrete tones. $\psi$ is the angle from the upstream jet axis to the observer centered about the nozzle exit. 


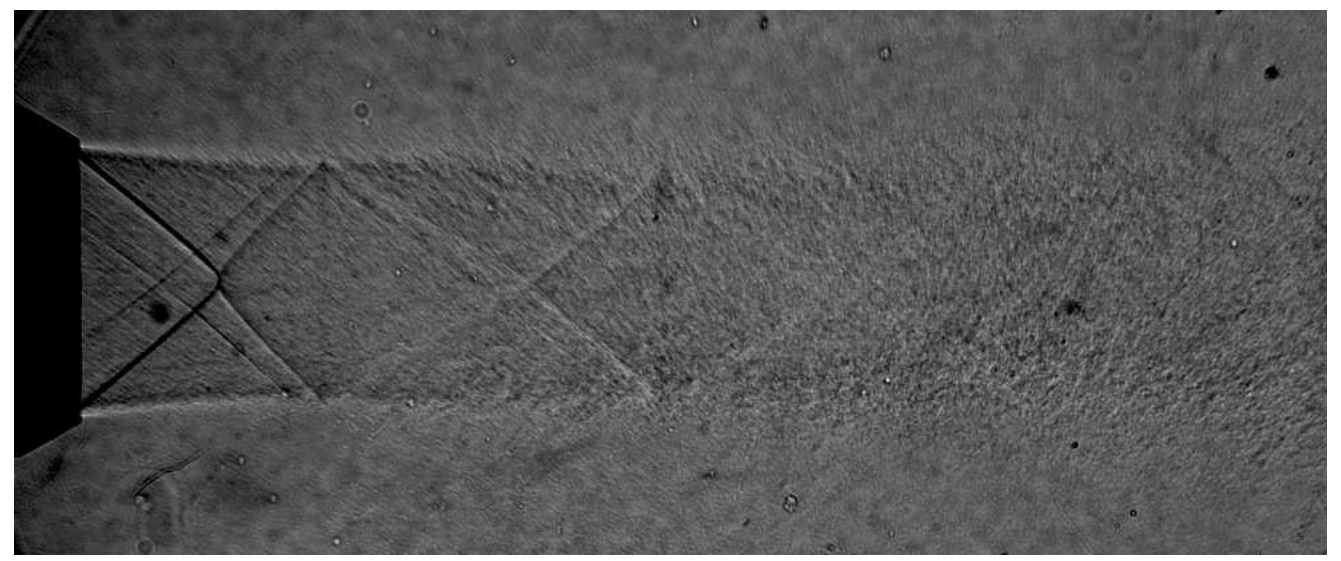

Figure 2. A schlieren of an over-expanded jet with fully expanded Mach number 1.60 from NASA Glenn Research Center courtesy of Zaman et al. ${ }^{14}$ The oblique shock waves and expansion waves are apparent within the jet plume.

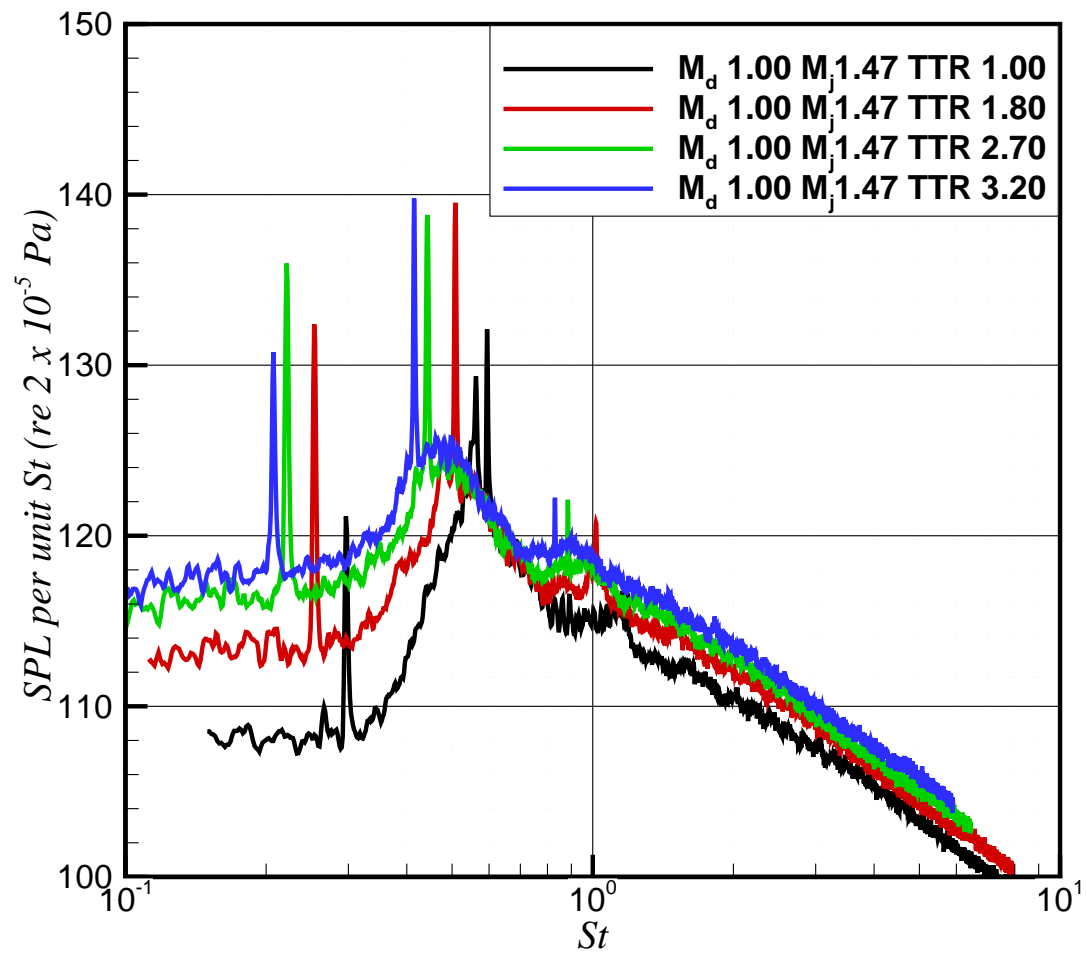

Figure 3. Spectra for a $M_{d}=1.00, M_{j}=1.47$, and $D=0.0127 \mathbf{~ m}$ jet at $R / D=100$ and $\psi=90$ degrees. Four jet $T T R$ spectra are shown at $1.00, \mathbf{1 . 8 0}, \mathbf{2 . 7 0}$, and 3.20 . Increasing the temperature causes the $B B S A N$ intensity to saturate. 

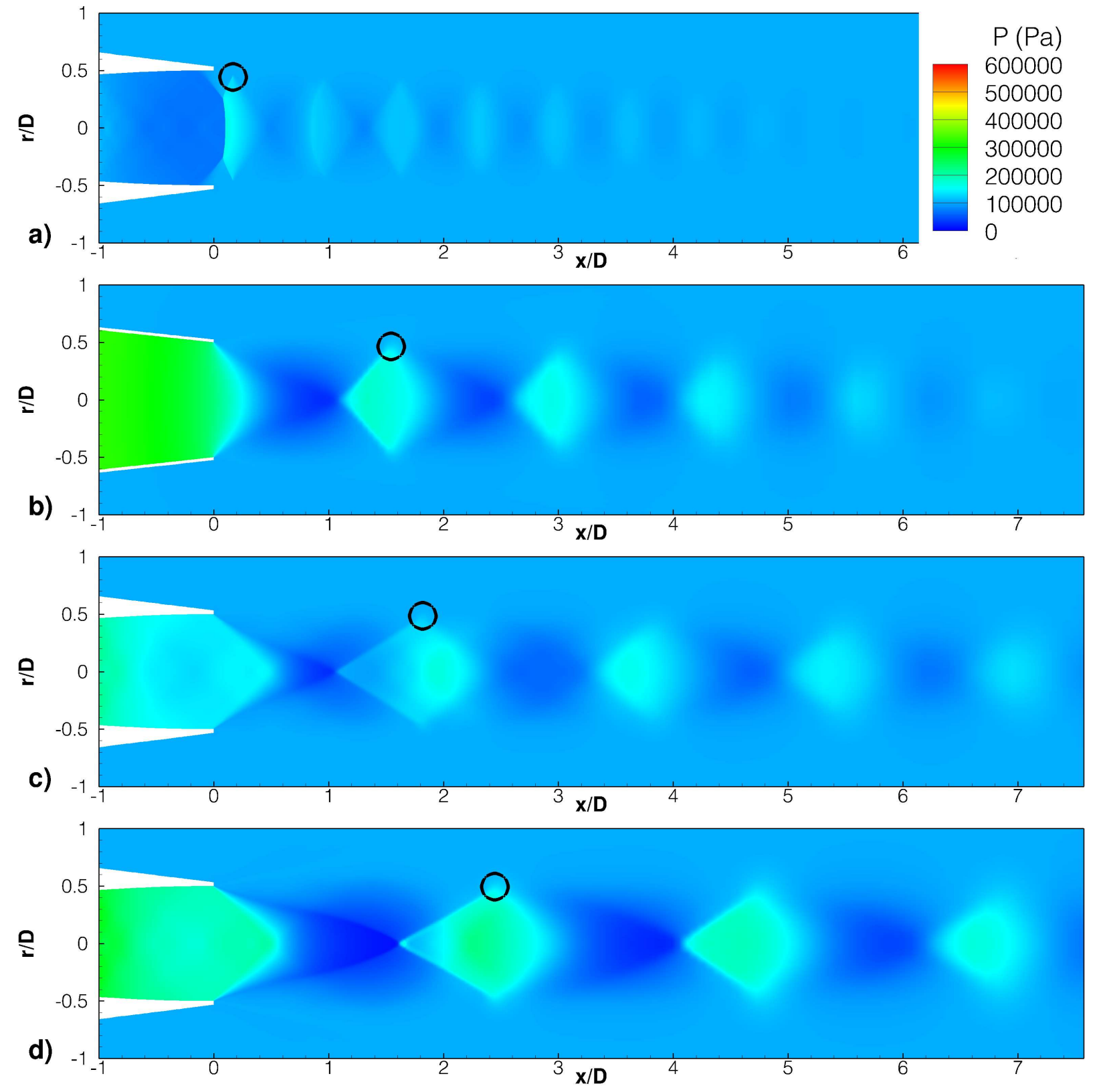

Figure 4. Contours of absolute pressure $(\mathrm{Pa})$ of the four jet families studied. The circles represent locations of the first oblique shock wave shear layer interaction. Flow-field data is extracted as a function of $T T R$ at these locations. The jet conditions shown are, a) $\left.M_{d}=1.50, M_{j}=1.20, T T R=1.00, \mathbf{b}\right) M_{d}=1.00, M_{j}=1.50$, $T T R=1.00$, c) $M_{d}=1.50, M_{j}=1.70, T T R=1.00$, d) $M_{d}=1.50, M_{j}=1.90, T T R=1.00$. 

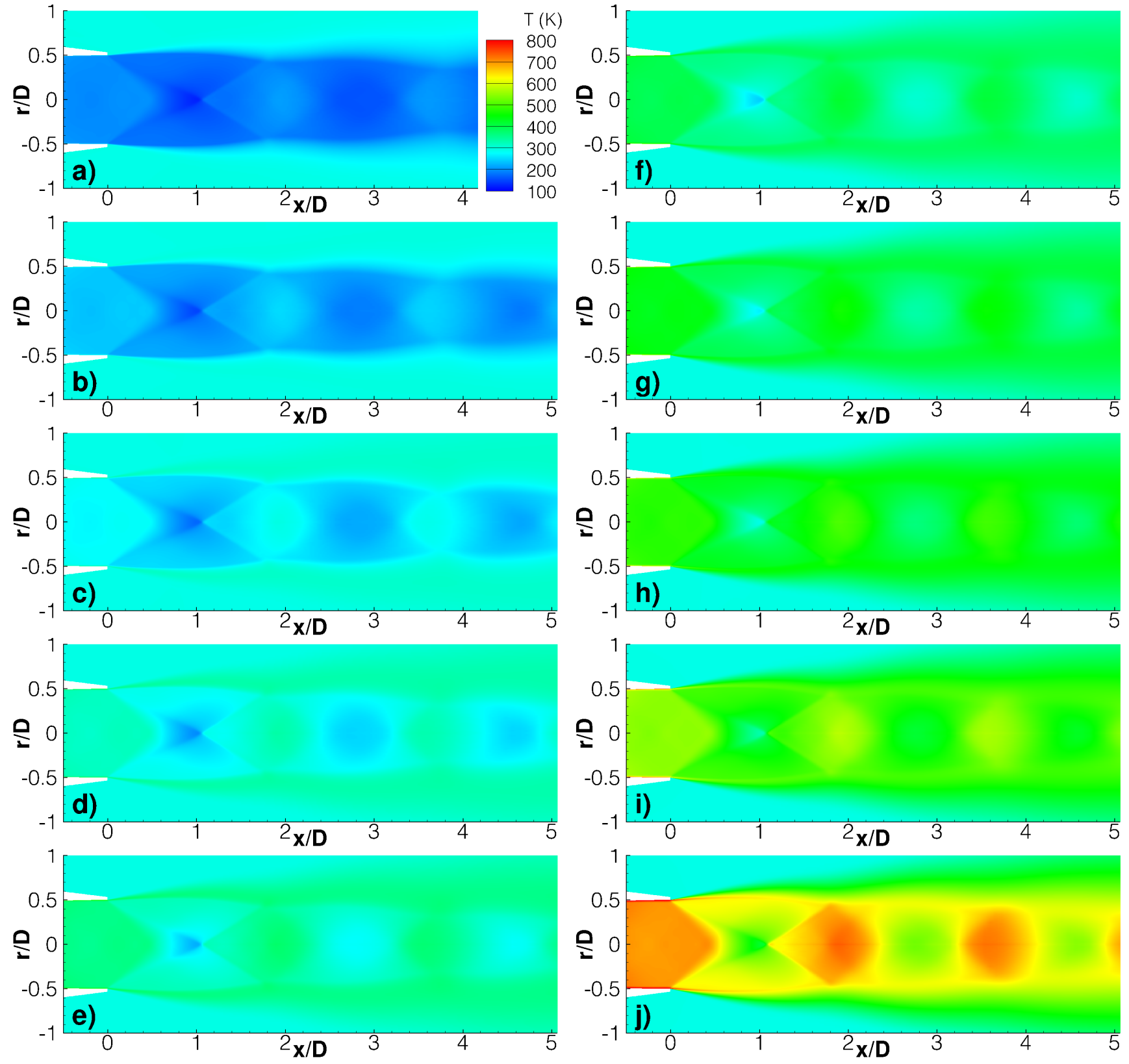

Figure 5. Contours of static temperature of select steady RANS solutions at $M_{d}=1.50, M_{j}=1.70$, and a) $T T R=1.00, \mathbf{b}) T T R=1.30, \mathbf{c}) T T R=1.50, \mathbf{d}) T T R=1.70, \mathbf{e}) T T R=1.90, \mathbf{f}) T T R=2.10, \mathbf{g}) T T R=2.30, \mathbf{h})$ $T T R=2.50$, i) $T T R=3.00, \mathbf{j}) T T R=3.50$ 


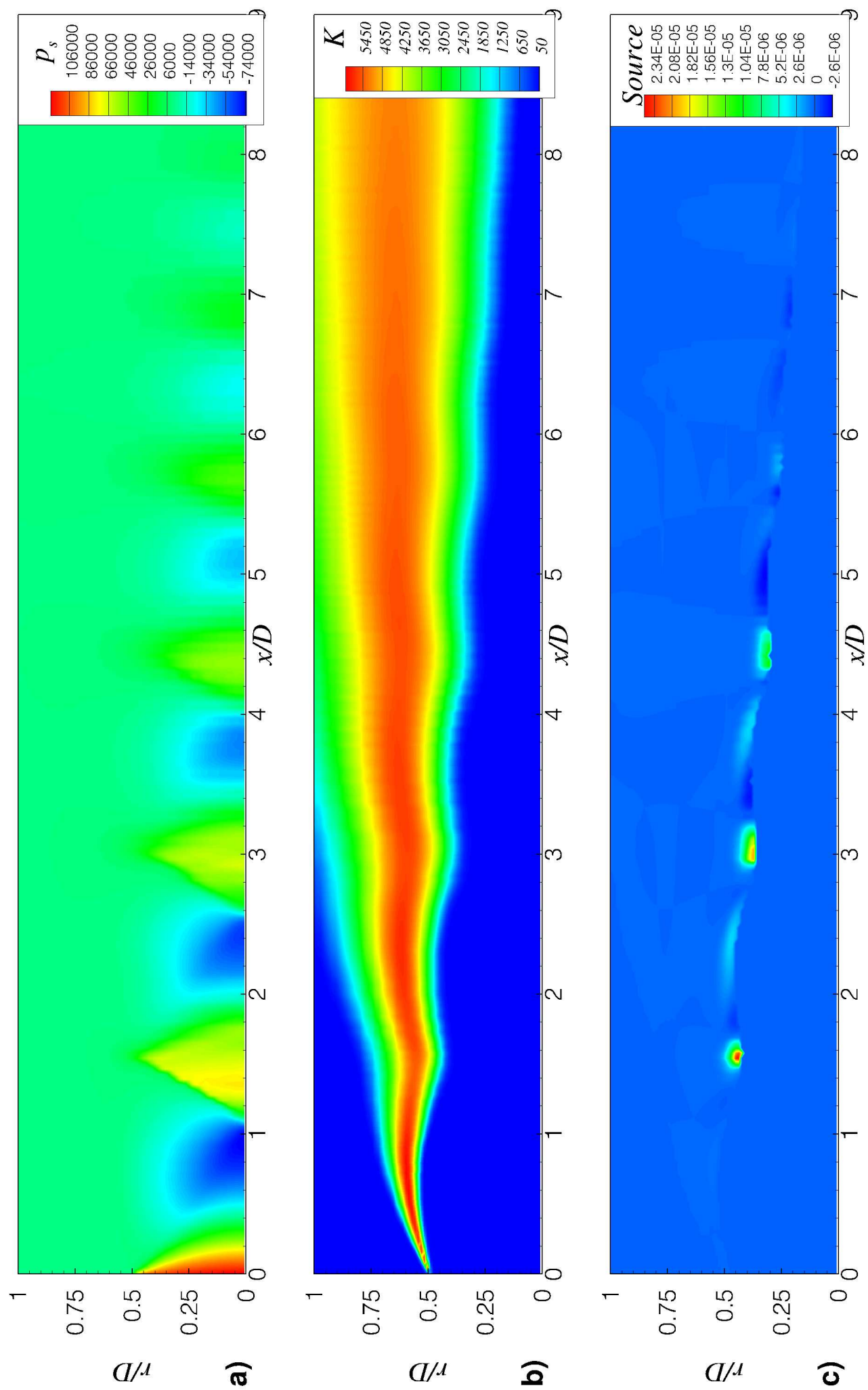

Figure 6. A $M_{d}=1.00, M_{j}=1.50, T T R=1.00, D=0.0254 \mathbf{m}$ jet produces contours of, a) shock pressure, b) turbulent kinetic energy, $K, \mathrm{c}$ ) integrand of the model of Morris and Miller ${ }^{27}$ at the sideline location at $R / D=100$ and peak BBSAN frequency (contours of BBSAN source strength). Note in c), the maximum BBSAN source occurs at the location where the shock wave shear layer interactions occur. 


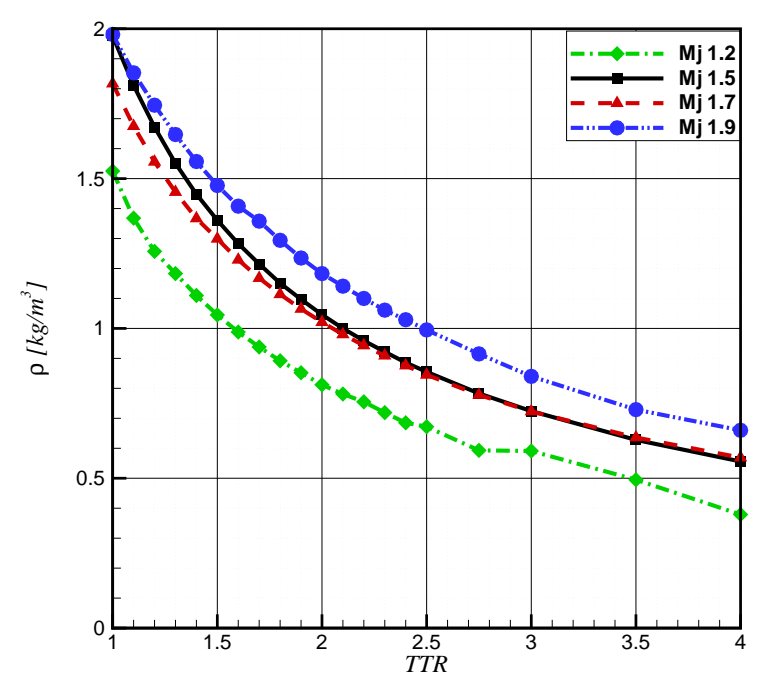

(a) Static density.

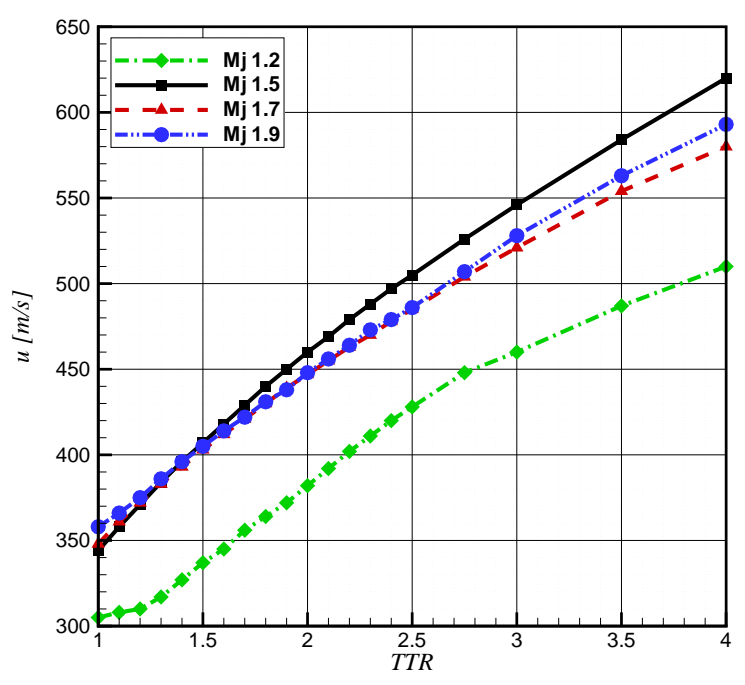

(b) Streamwise velocity component.

Figure 7. Variation of static density and the streamwise velocity component as a function of $T T R$ located at the first shock wave shear layer interaction.

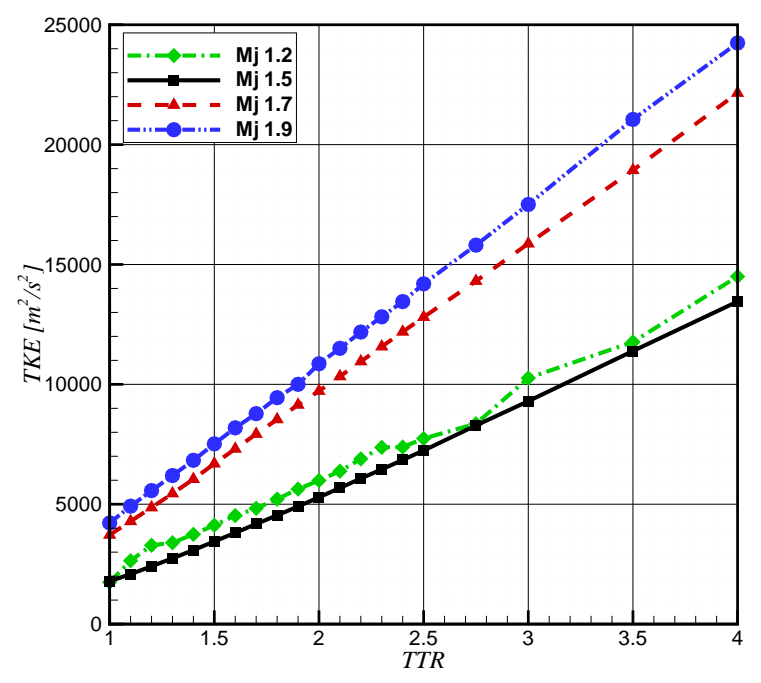

(a) Turbulent Kinetic Energy.

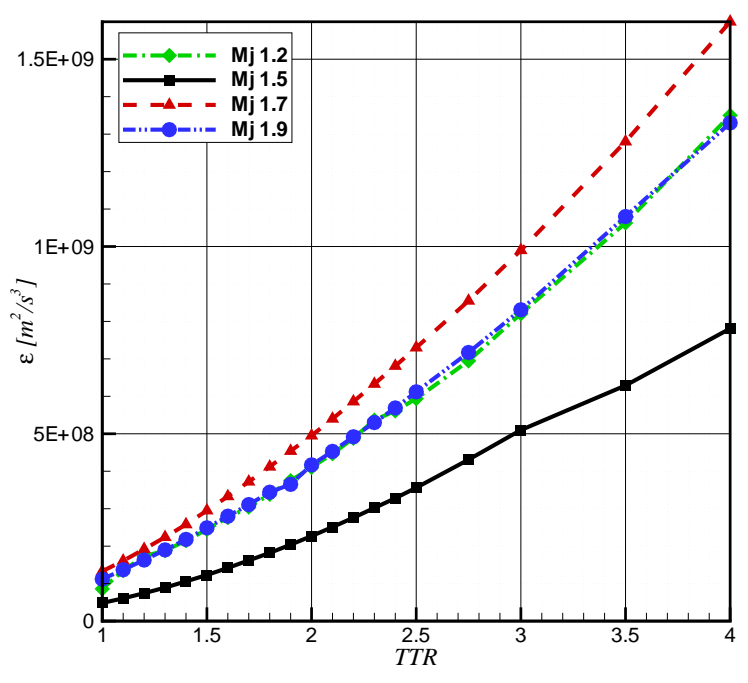

(b) Dissipation.

Figure 8. Variation of turbulent kinetic energy and dissipation as a function of $T T R$ located at the first shock wave shear layer interaction. 


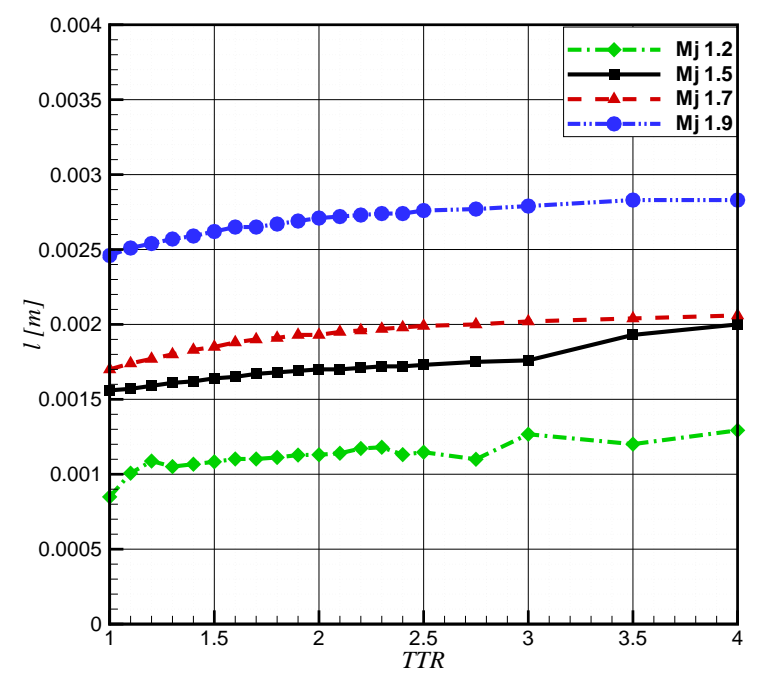

(a) Integral length scale in the streamwise direction.

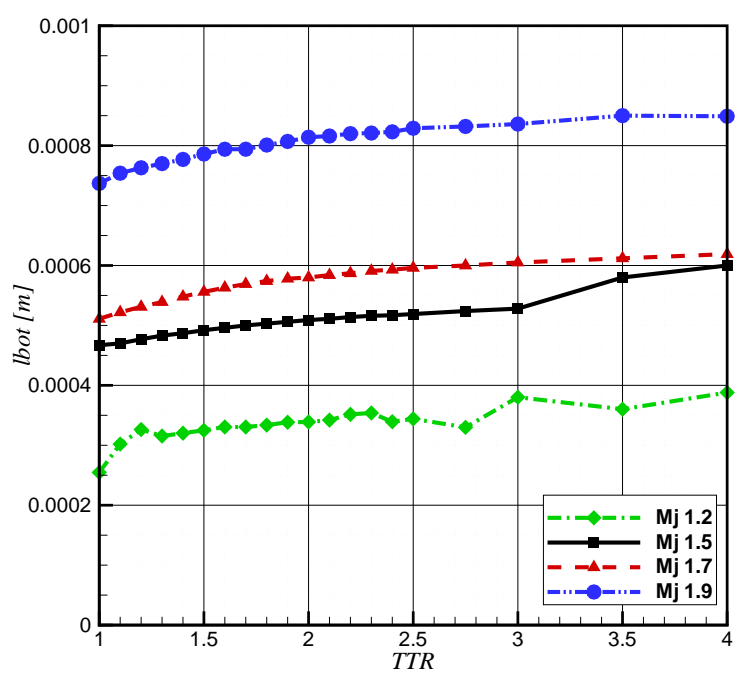

(b) Integral length scale in the cross-stream direction.

Figure 9. Variation of streamwise integral length scale and cross-stream integral length scale as a function of $T T R$ located at the first shock wave shear layer interaction.

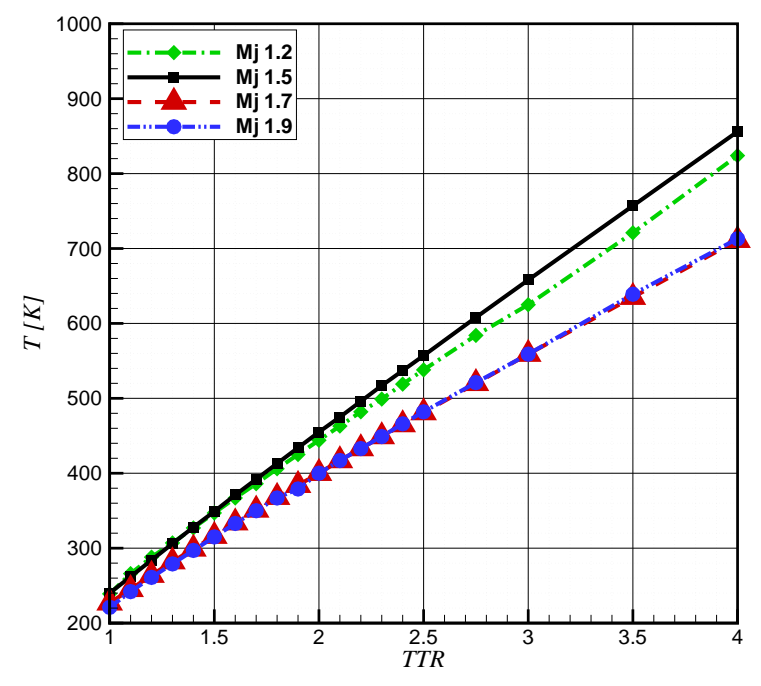

(a) Static temperature

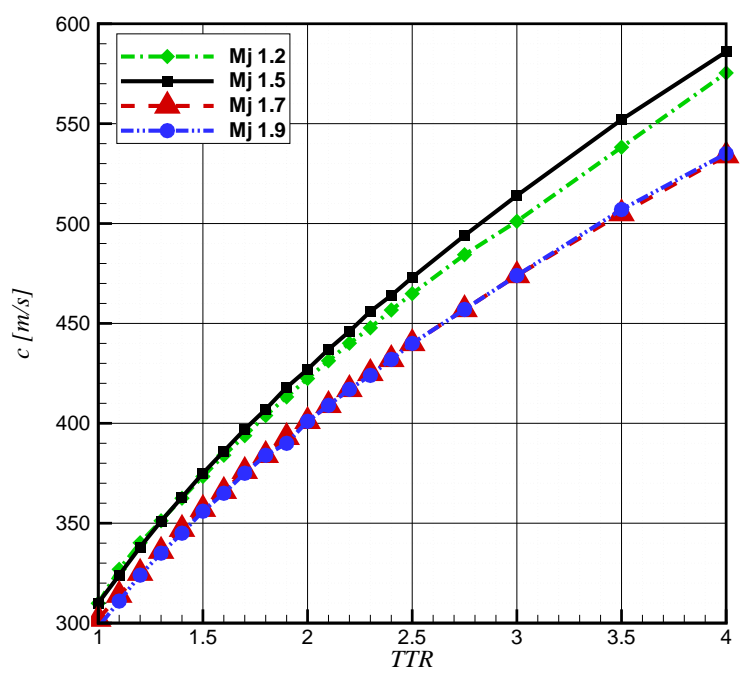

(b) Local speed of sound.

Figure 10. Variation of static temperature and local speed of sound as a function of $T T R$ located at the first shock wave shear layer interaction. 


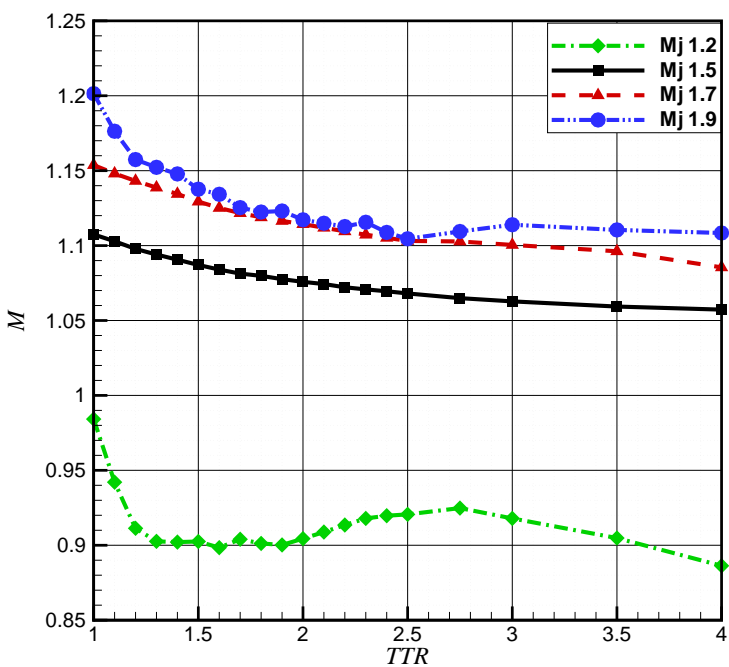

(a) Mach number referenced to local speed of sound.

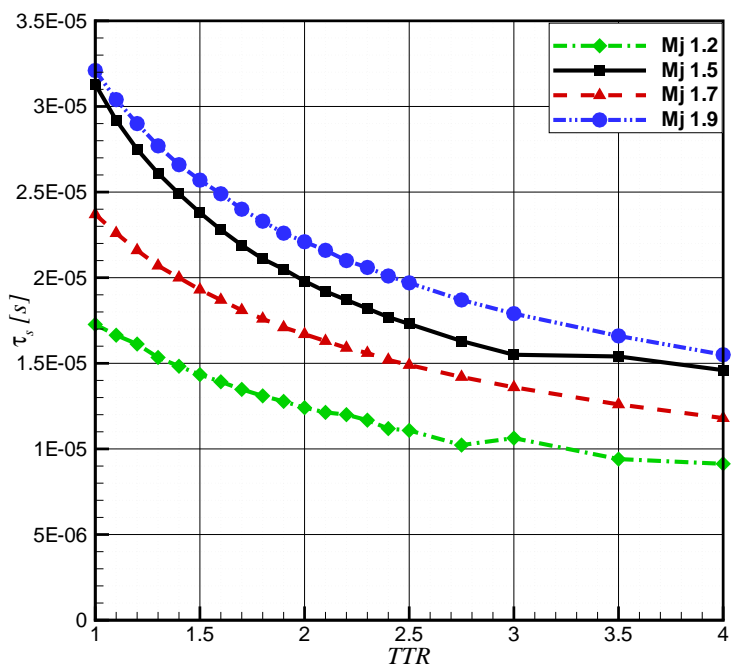

(b) Integral time scale.

Figure 11. Variation of the Mach number and the integral time scale as a function of $T T R$ located at the first shock wave shear layer interaction.

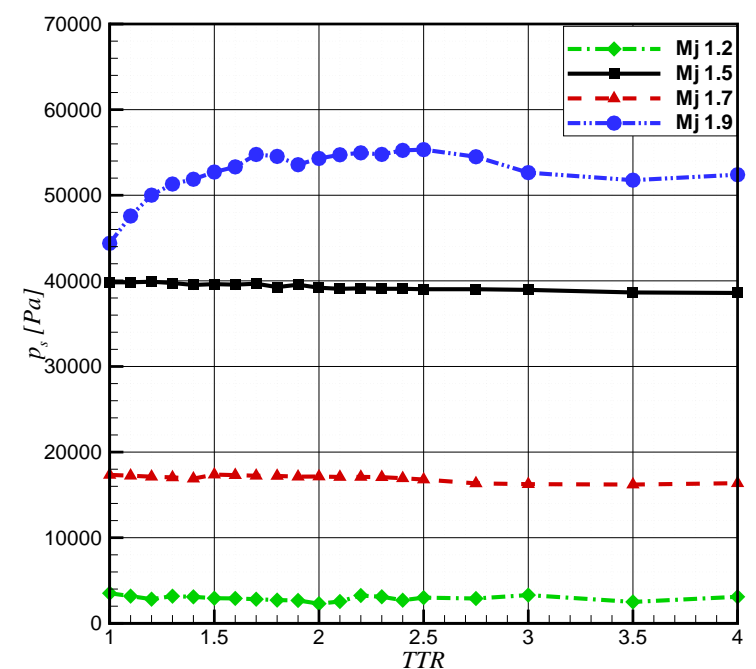

(a) Shock pressure.

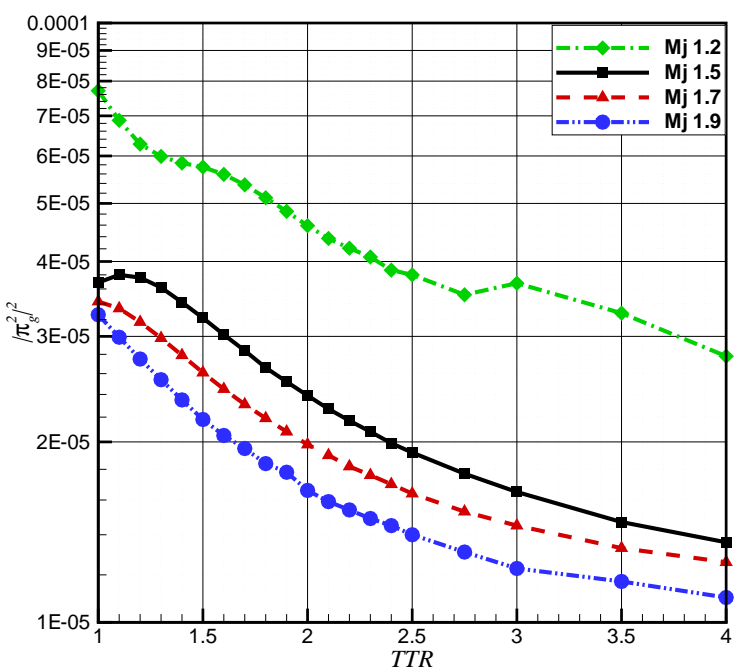

(b) Second component of the vector Green's function of the LEE for the pressure term.

Figure 12. Variation of the shock pressure and the second component of the vector Green's function of the LEE for the pressure term as a function of $T T R$ located at the first shock wave shear layer interaction. 


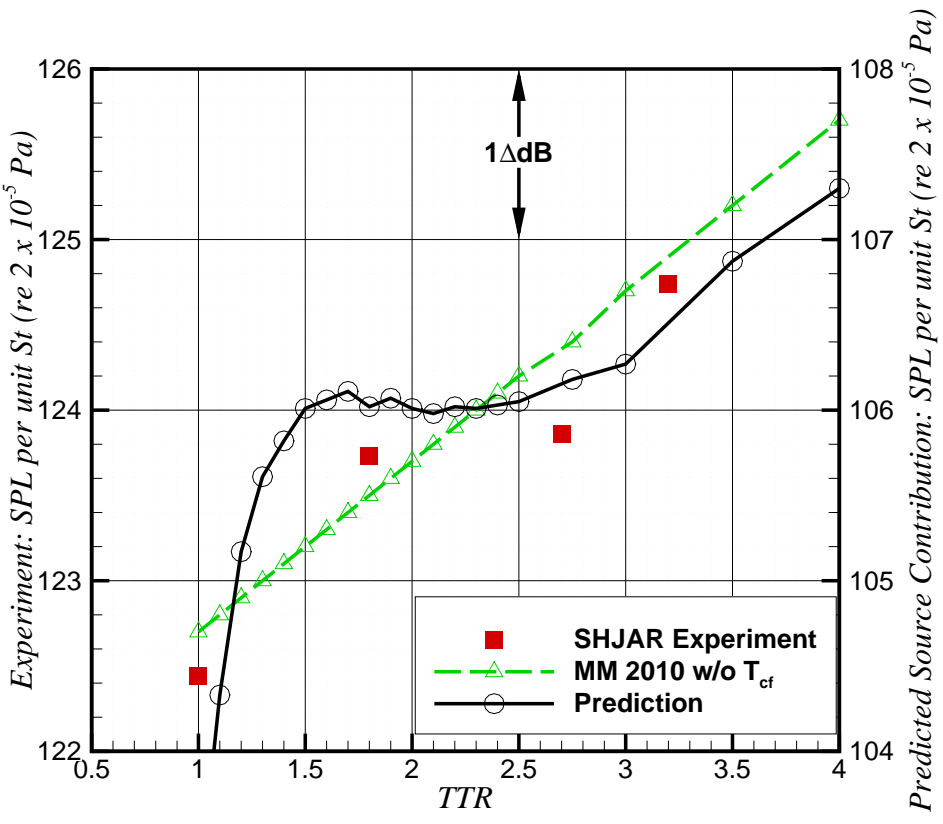

Figure 13. Variation of maximum BBSAN intensity from a $M_{d}=1, M_{j}=1.5$ (experiment performed at $\left.M_{j}=1.469\right)$ jet relative to increasing $T T R$. The observer is located at $R / D=100$ and $\psi=90$ degrees. The prediction of Eq. 26 is the contribution of a single shock wave shear layer interaction and is referenced to the right $y$-axis scale. Experimental data is referenced to the left $y$-axis scale. Note the scale between the prediction and experiment are the same.

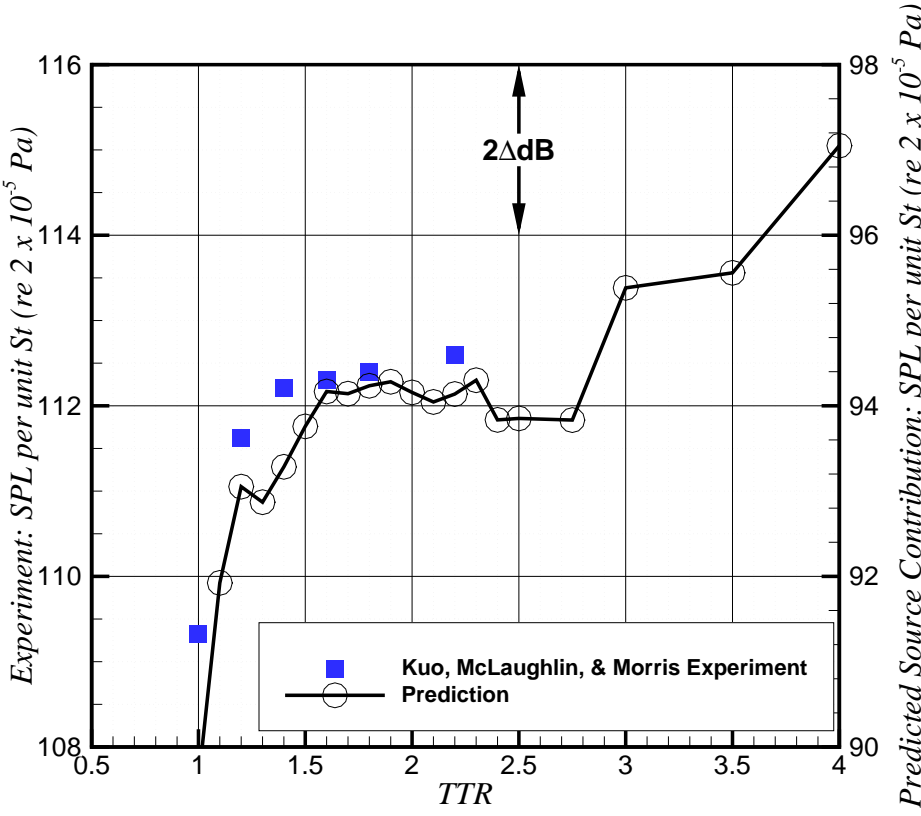

Figure 14. Variation of maximum BBSAN intensity from a $M_{d}=1.5, M_{j}=1.2$ jet relative to increasing $T T R$. The observer is located at $R / D=100$ and $\psi=90$ degrees. The prediction of Eq. 26 is the contribution of a single shock wave shear layer interaction and is referenced to the right $y$-axis scale. Experimental data is referenced to the left $y$-axis scale. Note the scale between the prediction and experiment are the same. 


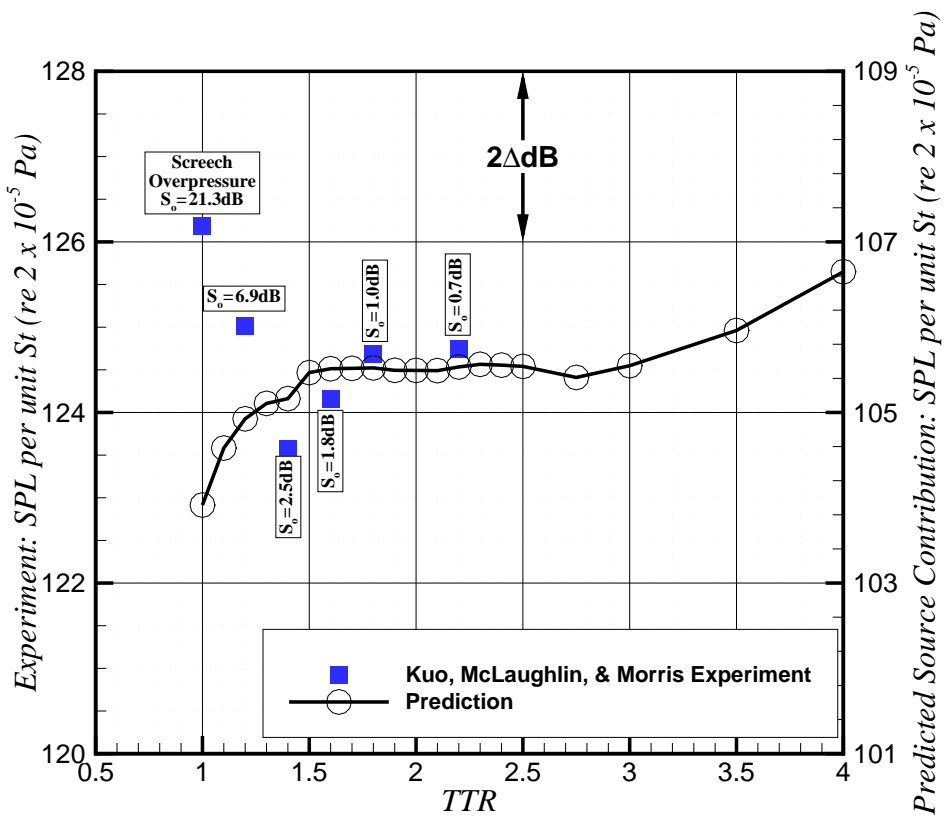

Figure 15. Variation of maximum BBSAN intensity from a $M_{d}=1.5, M_{j}=1.7$ jet relative to increasing $T T R$. The observer is located at $R / D=100$ and $\psi=90$ degrees. The prediction of Eq. 26 is the contribution of a single shock wave shear layer interaction and is referenced to the right $y$-axis scale. Experimental data is referenced to the left $y$-axis scale. Note the scale between the prediction and experiment are the same.

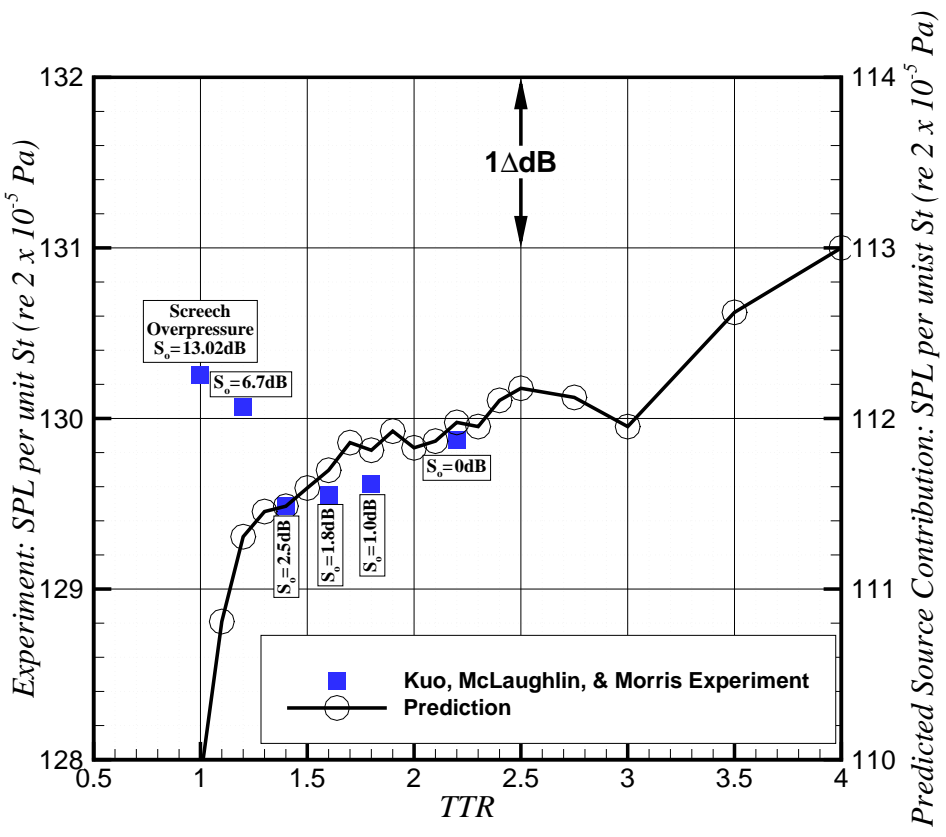

Figure 16. Variation of maximum BBSAN intensity from a $M_{d}=1.5, M_{j}=1.9$ jet relative to increasing $T T R$. The observer is located at $R / D=100$ and $\psi=90$ degrees. The prediction of Eq. 26 is the contribution of a single shock wave shear layer interaction and is referenced to the right $y$-axis scale. Experimental data is referenced to the left $y$-axis scale. Note the scale between the prediction and experiment are the same. 